\title{
A tumor-intrinsic PD-L1/NLRP3 inflammasome signaling pathway drives resistance to anti-PD-1 immunotherapy
}

\author{
Balamayoora Theivanthiran, ${ }^{1}$ Kathy S. Evans, ${ }^{1}$ Nicholas C. DeVito, ${ }^{1}$ Michael Plebanek, ${ }^{1}$ Michael Sturdivant, ${ }^{1}$ Luke P. Wachsmuth, \\ April K.S. Salama, ${ }^{1}$ Yubin Kang, ${ }^{2}$ David Hsu, ${ }^{1}$ Justin M. Balko, ${ }^{3}$ Douglas B. Johnson, ${ }^{3}$ Mark Starr, ${ }^{1}$ Andrew B. Nixon, ${ }^{1}$ \\ Alisha Holtzhausen, ${ }^{4}$ and Brent A. Hanks ${ }^{1,5}$ \\ 'Division of Medical Oncology and 2Division of Hematologic Malignancies and Cellular Therapy, Department of Medicine, Duke Cancer Institute, Durham, North Carolina, USA. ${ }^{3}$ Department of Medicine, \\ Vanderbilt University Medical Center, Nashville, Tennessee, USA. ${ }^{4}$ Lineberger Comprehensive Cancer Center, University of North Carolina at Chapel Hill, Chapel Hill, North Carolina, USA. ${ }^{5}$ Department of \\ Pharmacology and Cancer Biology, Duke University, Durham, North Carolina, USA
}

\begin{abstract}
An in-depth understanding of immune escape mechanisms in cancer is likely to lead to innovative advances in immunotherapeutic strategies. However, much remains unknown regarding these mechanisms and how they impact immunotherapy resistance. Using several preclinical tumor models as well as clinical specimens, we identified a mechanism whereby $\mathrm{CD8}{ }^{+} \mathrm{T}$ cell activation in response to programmed cell death 1 (PD-1) blockade induced a programmed death ligand 1/NOD-, LRR-, and pyrin domain-containing protein 3 (PD-L1/NLRP3) inflammasome signaling cascade that ultimately led to the recruitment of granulocytic myeloid-derived suppressor cells (PMN-MDSCs) into tumor tissues, thereby dampening the resulting antitumor immune response. The genetic and pharmacologic inhibition of NLRP3 suppressed PMN-MDSC tumor infiltration and significantly augmented the efficacy of anti-PD-1 antibody immunotherapy. This pathway therefore represents a tumor-intrinsic mechanism of adaptive resistance to anti-PD-1 checkpoint inhibitor immunotherapy and is a promising target for future translational research.
\end{abstract}

\section{Introduction}

Despite the significant impact that checkpoint inhibitor immunotherapies have generated in clinical oncology, the majority of patients with cancer still do not benefit from this treatment modality (1). It is widely believed that a more intimate understanding of the underlying mechanisms driving cancer immunotherapy resistance will lead to the discovery and development of innovative strategies to augment the efficacy of immunotherapy, while expanding the patient population capable of benefitting from these agents $(2,3)$. However, our understanding of the mechanisms driving both primary and secondary immunotherapy resistance remains incomplete.

There is an extensive body of literature describing the inhibitory role of myeloid-derived suppressor cells (MDSCs) in the generation of adaptive $\mathrm{T}$ cell immunity $(4,5)$. These data are

Conflict of interest: BAH receives research funding from Merck \& Co., Tempest Therapeutics, Leap Therapeutics, the Agency for Science, Technology, and Research Singapore, and AstraZeneca. AKSS receives research funding from Bristol-Myers Squibb, Celldex Therapeutics, Dynavax Technologies, Genentech, ImmunoCore, and Merck \& Co. JMB receives research support from Genentech, Bristol-Myers Squibb, and Incyte. DBJ receives research funding from Bristol-Myers Squibb and Incyte and serves on the scientific advisory boards for Array BioPharma, Bristol-Myers Squibb, Incyte, Merck \& Co., and Novartis.

Copyright: (5) 2020, American Society for Clinical Investigation.

Submitted: August 28, 2019; Accepted: January 29, 2020; Published: April 13, 2020

Reference information: J Clin Invest. 2020;130(5):2570-2586.

https://doi.org/10.1172/JCl133055. consistent with additional studies that have correlated elevated circulating MDSC levels with poor clinical responses to both anticytotoxic T lymphocyte antigen 4 (anti-CTLA-4) and anti-programmed cell death 1 (anti-PD-1) antibody $(\mathrm{Ab})$ immunotherapy in patients with advanced melanoma $(6,7)$. MDSCs have been shown to undergo chemotaxis toward tumor beds via chemokine gradients generated by the developing tumor. In particular, migration of the granulocytic subset of MDSCs (PMN-MDSCs) relies primarily on the chemokine receptor CXCR2 and several of its cognate ligands, including CXCL5 (8). Additional work has shown that CXCR2 blockade enhances the efficacy of anti-PD-1 $\mathrm{Ab}$ immunotherapy in models of both pancreatic cancer and sarcoma (8-10). These findings suggest that this immunosuppressive cell population plays a critical role in determining the outcome for patients with cancer undergoing anti-PD-1 Ab therapy. However, the exact mechanism by which MDSCs interfere with the development of antitumor immunity in response to checkpoint inhibitor immunotherapy remains unclear.

It is well known that the immune system is composed of many negative feedback inhibitory pathways that serve to suppress the development of overzealous immune responses to avoid autoimmune pathology. Similar mechanisms are likely to serve as the molecular underpinnings for the development of adaptive resistance to anti-PD-1 Ab immunotherapy and represent key pathways of interest for the future development of improved combinatorial immunotherapy strategies (11). Indeed, recent studies have demonstrated that both $\mathrm{CD}^{+} \mathrm{T}$ cell-dependent and IFN-depen- 
dent upregulation of CSF1 in melanoma and CD38 in lung cancer promote adaptive resistance to anti-PD-1 checkpoint blockade $(12,13)$. These observations are reminiscent of IFN-dependent upregulation of the immunoregulatory enzyme indoleamine 2,3-dioxygenase (IDO), which serves to reestablish immune tolerance in response to cytolytic T cell activity $(14,15)$. Although a recent study has implicated tumor expression of CXCL1 and the recruitment of PMN-MDSCs as key factors that mediate against tumor $\mathrm{T}$ cell infiltration, a role for CXCR2-dependent chemokines in the generation of adaptive resistance to anti-PD-1 Ab immunotherapy has not been described (16).

Previous work, including our own, has demonstrated the Wnt5a ligand to be associated with tumor progression, immune evasion, and immunotherapy resistance (17-19). Interestingly, TLR4 signaling regulates Wnt5a expression in myeloid cells and has also been associated with tumor progression in a variety of cancer types $(20,21)$.

Reports of tumor-intrinsic signaling pathways induced by programmed death ligand 1 (PD-L1) have emerged, linking PD-L1 with the promotion of epithelial-to-mesenchymal transition (EMT), stimulation of the mTOR/AKT antiapoptotic pathway, as well as inhibition of IFN-dependent apoptosis (22-24). Whereas each of these pathways may be protumorigenic, there are no known associations between PD-L1 and the induction of adaptive resistance to anti-PD-1 Ab immunotherapy via the stimulation of tumor-intrinsic signaling pathways. Many groups have described the role of NOD-, LRR-, and pyrin domain-containing protein 3 (NLRP3) as a sensor for pathogen-derived danger signals by antigen-presenting cells in the innate immune system, however, relatively little is known about the contribution of NLRP3 to tumorigenesis, and its role in modulating tumor responses to immunotherapy has not been explored (25).

Here, we describe a pathway that mechanistically links the upregulation of PD-L1 with the promotion of PMN-MDSC recruitment to the tumor bed in response to anti-PD-1 blockade and demonstrate that the inhibition of this process substantially enhances responses to checkpoint inhibitor immunotherapy (Figure $1 \mathrm{~A})$. We provide further evidence to support the existence of this pathway in patients with cancer.

\section{Results}

Anti-PD-1 Ab immunotherapy induces the recruitment of PMNMDSCs. We have found that the autochthonous $\mathrm{BRAF}^{\mathrm{V} 600 \mathrm{E}} \mathrm{PTEN}^{-/}$ melanoma model exhibits a transient response to anti-PD- $1 \mathrm{Ab}$ immunotherapy followed by eventual escape and progression. We harvested these melanoma tissues following anti-PD-1 Ab escape as well as after IgG isotype control Ab therapy and performed differential whole transcriptomic sequencing analysis. This study revealed the upregulation of 51 genes in anti-PD- $1 \mathrm{Ab}$-treated tumor tissues using a fold-change cutoff of $2.0(P<0.05)$. Of these genes, two CXCR2 ligands, $C x c l 5$ (3.75-fold, $\left.P=8.88 \times 10^{-6}\right)$ and $\mathrm{Cxcl3}$ (3.49-fold, $P=0.002)$, were found in the top 7 upregulated genes, whereas $C x c l 2$ was also noted to be upregulated by 3.63-fold $(P=0.146)$. These gene expression changes occurred concurrently with enhanced expression of the proinflammatory proteins S10Oa 8 (2.27-fold, $\left.P=1.61 \times 10^{-10}\right)$ and S100a9 (2.27-fold, $\left.P=3.37 \times 10^{-11}\right)$ as well as Arg1 (1.45-fold, $P=1.95 \times 10^{-6}$ ) (Figure
1B). We repeated the above experiment using a serial tissue biopsy approach coupled with quantitative real-time PCR (qRT-PCR) gene expression analysis, which confirmed a time-dependent increase in the expression of Cxcl2, Cxcl5, Cxcr2, Ly6g and the myeloid marker S100a9 during the course of anti-PD-1 Ab therapy relative to those tumors treated with an IgG isotype $\mathrm{Ab}$ (Figure 1C and Supplemental Figure 1A; supplemental material available online with this article; https://oi.org/10.1172/JCI133055DS1). Together, these data suggest that immunosuppressive PMNMDSC recruitment may correlate with suppression of cytolytic $\mathrm{T}$ cell activity along with anti-PD-1 Ab escape (Supplemental Figure 1A). To investigate this hypothesis, we evaluated resected melanoma tissue based on Gr-1 IHC as well as multiparameter flow cytometry, both of which confirmed a significant increase in infiltrating Gr- $1^{+}$and $\mathrm{CD}^{+} 5^{+} \mathrm{CD} 11 \mathrm{~b}^{+} \mathrm{Ly} 6 \mathrm{G}^{+} \mathrm{Ly} 6 \mathrm{C}^{\text {int }} \mathrm{F} 4 / 80^{-}$cell populations (PMN-MDSCs), respectively, with progression through anti-PD-1 $\mathrm{Ab}$ therapy (Figure 1, D and E). These findings were recapitulated in the Lewis lung carcinoma (LLC) lung cancer model, an orthotopic p53 Kras pancreatic cancer model, as well as in a humanized autologous patient-derived xenograft model of renal cell carcinoma (Supplemental Figure 1B). However, we did not observe any evidence of this effect following anti-CTLA-4 Ab therapy (Supplemental Figure 1C). qRT-PCR analysis of FACS-sorted PMNMDSCs from anti-PD-1 Ab-treated BRAF ${ }^{\mathrm{V} 600 \mathrm{E}} \mathrm{PTEN}^{-/-}$melanoma tissue confirmed that these cells expressed high levels of Cxcr2, Tnfa, S100a8, and S100a9 (Supplemental Figure 1D). Although we observed an increase in the expression of several CXCR2-dependent ligands following escape from anti-PD-1 Ab therapy, $\mathrm{CD}^{+} \mathrm{T}$ cell ablation studies demonstrated the CXCL5 chemokine to be particularly responsive to the induction of $\mathrm{CD} 8^{+} \mathrm{T}$ cell activation (Figure 1F). In addition, CXCL5 has previously been implicated in melanoma pathogenesis (26). Thus, we genetically silenced CXCL5 expression in a BRAF ${ }^{\mathrm{V} 600 \mathrm{E}} \mathrm{PTEN}^{-/-}$melanoma cell line, which effectively eliminated PMN-MDSC recruitment, enhanced tumor $\mathrm{CD}^{+} \mathrm{T}$ cell infiltration, and significantly increased the sensitivity of $\mathrm{BRAF}^{\mathrm{V} 600 \mathrm{E}} \mathrm{PTEN}^{-/-}$melanomas to anti-PD-1 Ab immunotherapy (Figure $1 G$ and Supplemental Figure 2). Further in vivo tumor studies using a pharmacological CXCR2 inhibitor (AZD5069) also significantly suppressed PMN-MDSC recruitment in response to anti-PD- 1 Ab therapy, enhanced $\mathrm{CD}^{+} \mathrm{T}$ cell tumor infiltration, and suppressed tumor progression in the autochthonous $\mathrm{BRAF}^{\mathrm{V} 600 \mathrm{E}} \mathrm{PTEN}^{-/}$melanoma model (Figure $1 \mathrm{H}$ ). Notably, we found the impact of AZD5069 to be more significant at later time points correlating with the period of PMN-MDSC influx into the tumor (Supplemental Figure 2D). Together, these data indicate that tumors exhibit an increase in CXCR2 ligandmediated PMN-MDSC recruitment to the tumor bed during their progression through anti-PD-1 Ab immunotherapy.

Wnt5a promotes CXCR2 ligand expression in response to anti$P D-1$ immunotherapy. The differential whole transcriptomic analysis of the autochthonous BRAF ${ }^{\mathrm{V} 600 \mathrm{E}} \mathrm{PTEN}^{-/-}$melanoma model subjected to anti-PD-1 Ab versus IgG isotype control Ab therapy demonstrated several genetic changes suggestive of enhanced Wnt ligand signaling during anti-PD-1 Ab escape (Supplemental Figure 3A). Additional analysis of transcriptomic data for patients with metastatic melanoma in the The Cancer Genome Atlas-SKCM (TCGA-SKCM) database also revealed a statistical- 
A

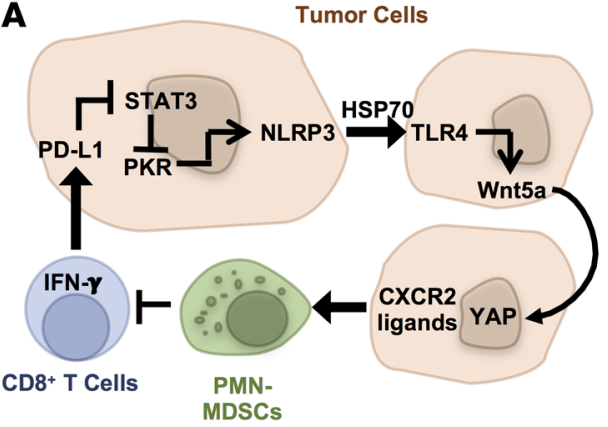

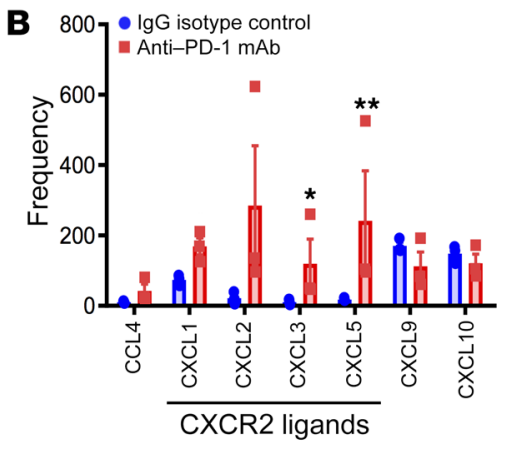

C
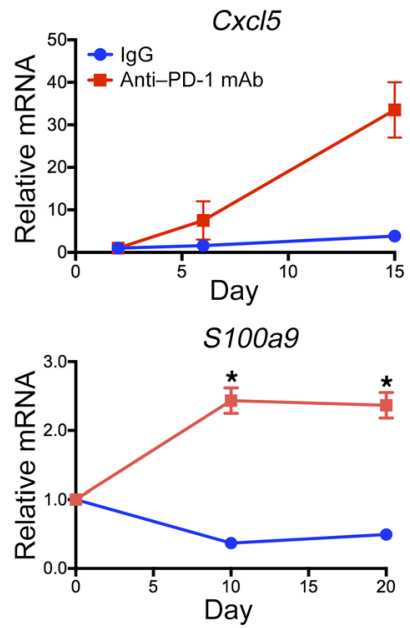

F

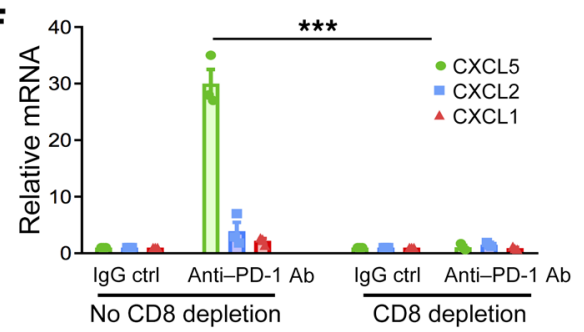

D

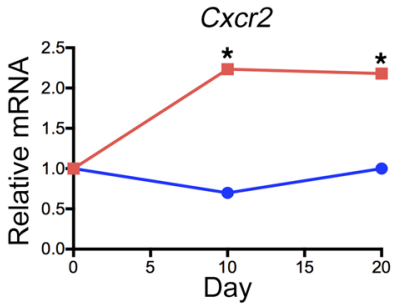

Ly6g

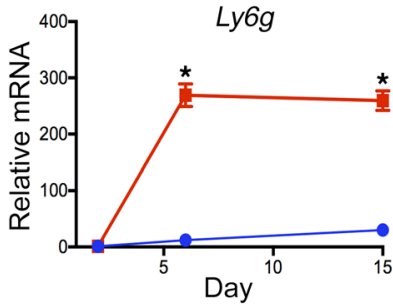

G

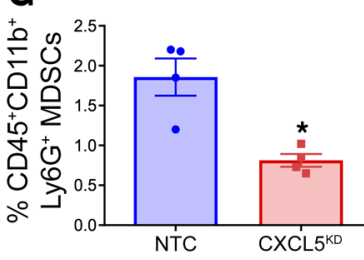

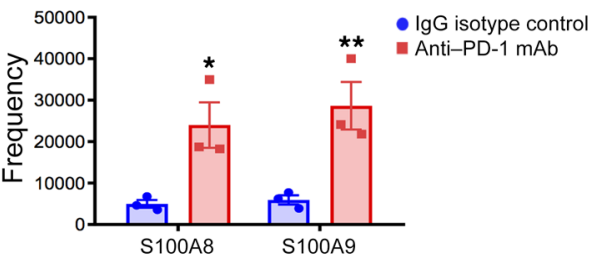

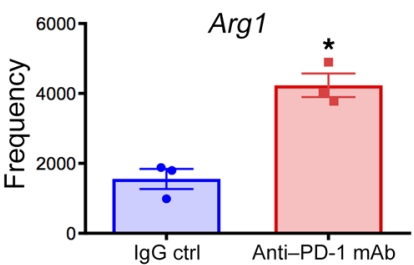

Gr-1
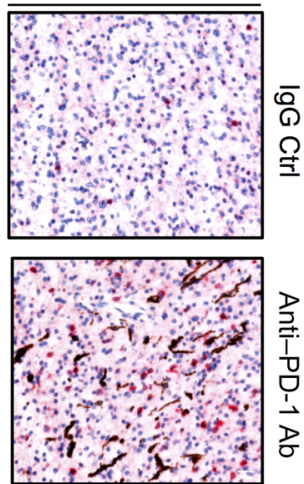
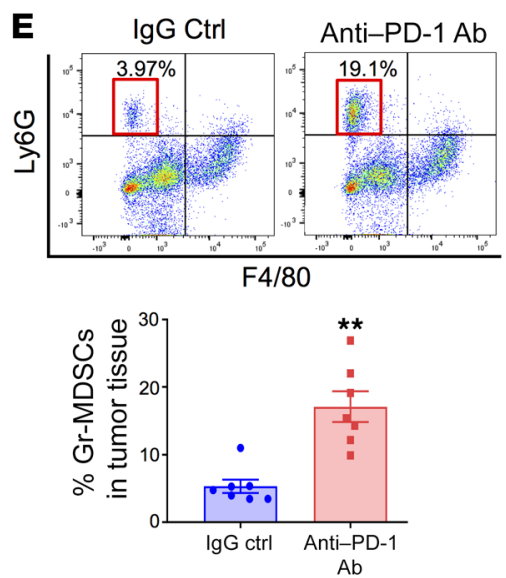
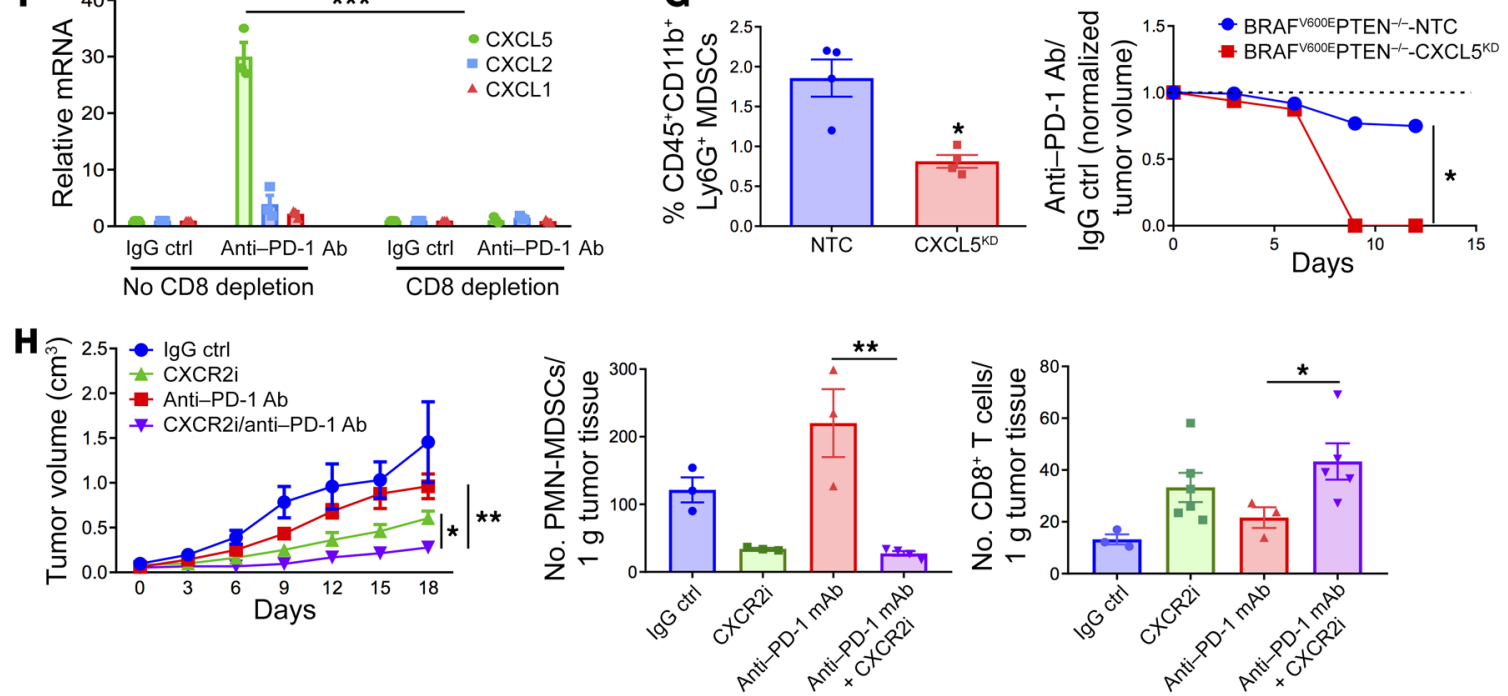

Figure 1. PMN-MDSC accumulation contributes to tumor progression following anti-PD-1 Ab immunotherapy. (A) Schematic overview of the adaptive resistance pathway. (B) RNA-Seq differential gene expression analysis of tumor tissues following treatment of the autochthonous BRAF ${ }^{\mathrm{V} 600 \mathrm{E}} \mathrm{PTEN}^{-/-}$melanoma model with anti-PD-1 Ab therapy versus IgG isotype control (Ctrl) $(n=3)$. (C) qRT-PCR analysis of target genes of interest in serial tumor fine-needle aspiration (FNA) biopsy specimens harvested from the transgenic BRAF ${ }^{\mathrm{V} 600 \mathrm{E}} \mathrm{PTEN}^{-1-}$ melanoma model treated with anti-PD-1 Ab versus IgG isotype control $(n=5)$. (D) Gr-1 immunohistochemical analysis of transgenic BRAF ${ }^{\mathrm{V} 600 \mathrm{E}} \mathrm{PTEN}^{-/-}$melanoma tissues following treatment with anti-PD-1 Ab versus IgC isotype control. Original magnification, $\times 40$. Gr-1 staining is shown in red. Images are representative of 3 tumors per group. (E) PMN-MDSC flow cytometric analysis of transgenic BRAF ${ }^{\mathrm{V} 600 \mathrm{E}} \mathrm{PTEN}^{-/-}$melanoma tissues following treatment with anti-PD-1 Ab versus IgG isotype control. PMN-MDSCs were defined as live+CD45+CD11b+Ly6C+'Ly6C intF4/80- cells. Shown are a representative flow dot plot and quantification graph of PMN-MDSC flow cytometric data $(n=5)$. (F) qRT-PCR analysis of CXCR2 ligands in BRAFV600E PTEN ${ }^{-1-}$ melanoma tissues treated with anti-PD-1 Ab following CD8 ${ }^{+}$T cell ablation in vivo $(n=3)$. (C) In vivo tumor study of BRAF ${ }^{\mathrm{V} 600 \mathrm{E}} \mathrm{PTEN}^{-/-}$melanoma genetically silenced for CXCL5. Quantitation of tumor-infiltrating PMN-MDSCs by flow cytometry is shown along with an in vivo tumor growth curve of CXCL5-silenced BRAF ${ }^{\mathrm{V} 600 \mathrm{E}} \mathrm{PTEN}^{-1-}$ melanoma versus BRAF $\mathrm{V}^{\mathrm{V} 600 \mathrm{P}} \mathrm{PTEN}^{-1-}$ NTC melanoma control tumors treated with anti-PD-1 Ab. Data were normalized to tumors treated with IgG isotype control $(n=5)$. (H) Combination treatment with anti-PD-1 Ab and CXCR2 inhibitor (CXCR2i) in an in vivo BRAF ${ }^{\mathrm{V} 600 \mathrm{E}} \mathrm{PTEN}^{-/-}$melanoma study $(n=5)$. Graphs show flow cytometric analysis of tumor-infiltrating PMN-MDSCs and live ${ }^{+}$ CD45 ${ }^{+} \mathrm{CD3}^{+} \mathrm{CD}^{+}$T cells. ${ }^{*} P<0.05$, ${ }^{*} P<0.005$, and ${ }^{* * *} P<0.0005$, by Student's $t$ test with Holm-Sidak post hoc correction for multiple comparisons (B, C, and $\mathbf{F}$ ), Student's $t$ test (E and $\mathbf{G}$ ), or 1-way ANOVA with Sidak's post hoc multiple comparisons test (H). See also Supplemental Figures 1, 2, and 5C. 
ly significant association between WNT5A and CXCL2, CXCL5, and CXCR2 gene expression (Figure 2A). Previous studies have shown that noncanonical Wnt ligands activate Yes-associated protein-dependent (YAP-dependent) signaling pathways, whereas YAP signaling has also been implicated in the migration of PMN-MDSCs into tumor tissues $(27,28)$. Consistent with these data, we performed whole-tissue Western blot analysis and found that resected melanoma tissues derived from the autochthonous $\mathrm{BRAF}^{\mathrm{V} 600 \mathrm{E}} \mathrm{PTEN}^{-/-}$model had enhanced Wnt5a expression, YAP stabilization, and increased CXCL5 expression in response to anti-PD-1 Ab therapy (Figure 2B). This enhanced level of CXCL5 expression by the tumor was also reflected by increased circulating plasma CXCL5 levels as determined by ELISA (Figure 2C). We performed further in vitro studies using qRT-PCR, which confirmed that recombinant Wnt5a (rWnt5A) induced Cxcl2, Cxcl5, and $\mathrm{Cxcl1}$ gene expression in the $\mathrm{BRAF}^{\mathrm{V} 600 \mathrm{E}} \mathrm{PTEN}^{-/-}$melanoma cell line (Figure 2D). Given these findings, we hypothesized that upregulation of Wnt5a results in enhanced CXCR2-dependent chemokine expression by triggering the YAP signaling pathway. Further experiments using the $\mathrm{BRAF}^{\mathrm{V} 600 \mathrm{E}} \mathrm{PTEN}^{-/}$melanoma cell line indeed showed that rWnt5a induced YAP stabilization, while also stimulating CXCL5 expression in a YAP-dependent manner (Figure 2E). To confirm the role of Wnt5a in the upregulation of CXCL5 in the BRAF ${ }^{\mathrm{V} 600 \mathrm{E}} \mathrm{PTEN}^{-/-}$melanoma cell line, qRT-PCR further revealed diminished CXCL5 expression in a Wnt5a-knockdown $\left(\right.$ Wnt5 ${ }^{\mathrm{KD}}$ ) BRAF ${ }^{\mathrm{V} 600 \mathrm{E}} \mathrm{PTEN}^{-/-}$melanoma cell line $\left(\mathrm{BRAF}^{\mathrm{V} 600 \mathrm{E}}\right.$ $\mathrm{PTEN}^{-/-}$Wnt5a $\left.{ }^{\mathrm{KD}}\right)$, an effect that could be rescued with the addition of rWnt5a (Figure $2 \mathrm{~F}$ and Supplemental Figure 3B). Additional studies using flow cytometry and IHC showed that BRAFV600E $\mathrm{PTEN}^{-/-}$Wnt5a ${ }^{\mathrm{KD}}$ tumors had reduced CXCL5 expression and an associated decrease in intratumoral PMN-MDSCs, respectively (Figure 2, G and H). On the basis of these data, we hypothesized that the previously observed recruitment of PMN-MDSCs to the tumor bed in response to anti-PD-1 Ab therapy would be eliminated in tumors silenced for Wnt5a expression. Indeed, PMN-MDSC recruitment to $\mathrm{BRAF}^{\mathrm{V} 600 \mathrm{E}} \mathrm{PTEN}^{-/-} \mathrm{Wnt}^{\mathrm{K}} \mathrm{a}^{\mathrm{KD}}$ tumors was significantly diminished relative to control $\mathrm{BRAF}^{\mathrm{V} 600 \mathrm{E}} \mathrm{PTEN}^{-/}$tumors in response to anti-PD-1 Ab therapy (Figure 2I). Consistent with an important role for PMN-MDSCs in driving immunotherapy resistance, we also found $\mathrm{BRAF}^{\mathrm{V} 600 \mathrm{E}} \mathrm{PTEN}^{-/-} \mathrm{Wnt}^{\mathrm{K}} \mathrm{a}^{\mathrm{KD}}$ tumors to be associated with increased $\mathrm{CD}^{+} \mathrm{T}$ cell infiltration and to respond more favorably to anti-PD-1 Ab therapy relative to control BRAF ${ }^{\mathrm{V} 600 \mathrm{E}} \mathrm{PTEN}^{-/-}$tumors (Figure 2J and Supplemental Figure $3 \mathrm{C})$. Altogether, these findings support a role for tumor-derived Wnt5a as an important mediator of CXCL5-dependent PMNMDSC recruitment to the tumor bed in response to anti-PD- $1 \mathrm{Ab}$ checkpoint inhibitor immunotherapy.

An autocrine HSP7O/TLR4 signaling pathway stimulates tumor Wnt5a release in response to anti-PD-1 immunotherapy. Given our previous data, we then investigated the underlying mechanism for Wnt5a upregulation in response to anti-PD-1 Ab therapy in the $\mathrm{BRAF}^{\mathrm{V} 600 \mathrm{E}} \mathrm{PTEN}^{-/-}$melanoma model. Prior studies have shown that TLR signaling modulates Wnt5a expression in macrophages (20). Heat shock proteins (HSPs) are known to bind and induce the activation of TLR signaling pathways (29). A review of the previous whole transcriptomic data analysis performed in the autochthonous BRAF ${ }^{\mathrm{V} 600 \mathrm{E}} \mathrm{PTEN}^{-/-}$melanoma model showed that anti-PD-1 Ab therapy results in increased expression of several genes associated with cellular stress, including a subset of HSPs (Figure 3A) (30). To investigate this further, we analyzed the secretome of resected $\mathrm{BRAF}^{\mathrm{V} 600 \mathrm{E}} \mathrm{PTEN}^{-/-}$melanoma tissues following escape from anti-PD-1 Ab immunotherapy, using liquid chromatography-tandem mass spectrometry (LC-MS/MS) and the stable isotope labeling with amino acids in cell culture (SILAC) technique coupled with azidohomoalanine (AHA) labeling (31). This work showed further evidence of increased release of HSPs, including HSP70, in those tumors that had escaped anti$\mathrm{PD}-1 \mathrm{Ab}$ immunotherapy relative to IgG isotype Ab control-treated tumors (Figure 3B). Consistent with these data, we also found that melanoma-bearing mice undergoing anti-PD- $1 \mathrm{Ab}$ treatment had increased circulating plasma HSP70 levels relative to levels in mice treated with an IgG isotype control Ab (Figure 3C). A qRT-PCR-based screen identified elevated levels of Tlr2 and Tlr4 expression by $\mathrm{BRAF}^{\mathrm{V} 600 \mathrm{E}} \mathrm{PTEN}^{-/-}$melanoma cells relative to other TLRs, and an analysis of TCGA database also revealed a compelling relationship between $W n t 5 a$ and TLR 2 andTLR 4 expression in human melanoma (Figure 3D and Supplemental Figure 4A). Consistent with this, we found that HSP70 stimulation of the $\mathrm{BRAF}^{\mathrm{V} 600 \mathrm{E}} \mathrm{PTEN}^{-/-}$melanoma cell line induced upregulation of Wnt5a expression in a dose-dependent manner, whereas pharmacologic inhibition of HSP70 suppressed autocrine stimulation of Wnt5a expression (Figure 3, E and F). Subsequent pharmacologic inhibitor and genetic silencing studies using a shRNA-expressing lentiviral vector revealed that HSP70 stimulation of melanoma Wnt5a expression was TLR4 dependent (Figure 3G and Supplemental Figure 4, B and C). Interestingly, these $\mathrm{BRAF}^{\mathrm{V} 600 \mathrm{E}} \mathrm{PTEN}^{-/-}$ melanoma cells genetically silenced for Tlr4 did not generate tumors in vivo following their implantation. Even with modest Tlr4 silencing using siRNA oligonucleotides, these melanomas exhibited diminished tumor growth, reduced Wnt5a and CXCL5 expression based on whole-tissue Western blot analysis, and a lower level of tumor-infiltrating PMN-MDSCs along with enhanced numbers of $\mathrm{CD}^{+} \mathrm{T}$ cells compared with control $\mathrm{BRAF}^{\mathrm{V} 600 \mathrm{E}} \mathrm{PTEN}^{-/-}$melanomas (Figure 3, H-J, and Supplemental Figure 4, D and E). To verify that HSP70 induction of the Wnt5a/CXCL5 signaling axis is not a specific phenomenon related to melanoma, we also repeated these experiments in a murine lung epithelial cell line with similar results (Supplemental Figure 4F). In summary, these data suggest that tumor HSP70 release in response to anti-PD-1 Ab treatment induces Wnt5a-mediated upregulation of CXCR2-dependent chemokine expression in tumor tissues.

$C D 8^{+} T$ cells drive the HSP7O/TLR4/Wnt5a/CXCL5 signaling axis in tumors. Since our data indicated that PMN-MDSC recruitment was induced by anti-PD-1 Ab therapy, we hypothesized that $\mathrm{CD}^{+} \mathrm{T}$ cells play an important role in triggering the HSP70/ TLR4-Wnt5a/CXCL5 signaling cascade. We therefore cocultured increasing numbers of OT- $1 \mathrm{~K}^{\mathrm{b}}$-SIINFEKL-specific CD8 ${ }^{+}$ T cells with a BRAF ${ }^{\mathrm{V} 600 \mathrm{E}} \mathrm{PTEN}^{-/-}$melanoma cell line engineered to express the OVA xenoantigen (BRAF ${ }^{\mathrm{V} 600 \mathrm{E}} \mathrm{OVA}$ ) and measured the soluble production of HSP7O (Supplemental Figure 5A). This approach showed a direct correlation between antigen-specific $\mathrm{CD}^{+} \mathrm{T}$ cells, tumor HSP70 secretion, and the induction of Wnt5a expression in vitro (Figure 4A). Further flow cytometric analysis of resected $\mathrm{BRAF}^{\mathrm{V} 600 \mathrm{E}} \mathrm{PTEN}^{-/-}$melanoma tissues also revealed 

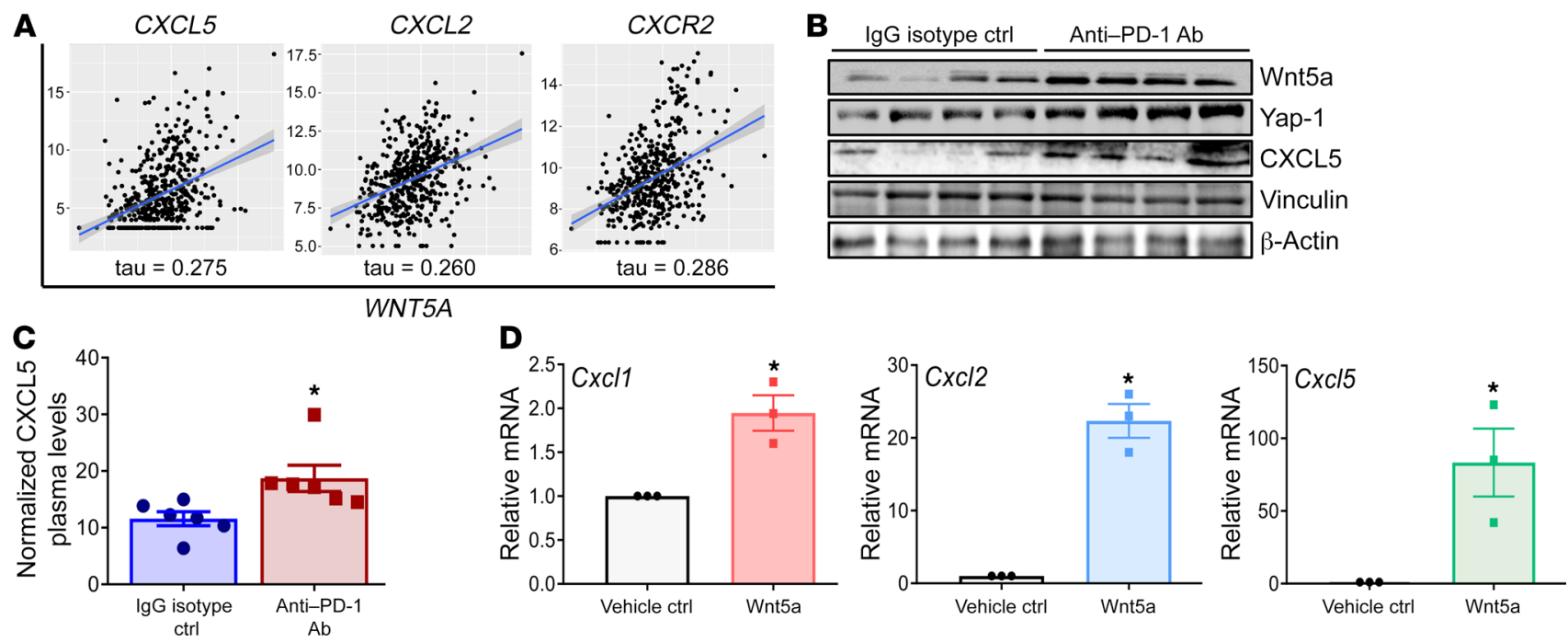

E
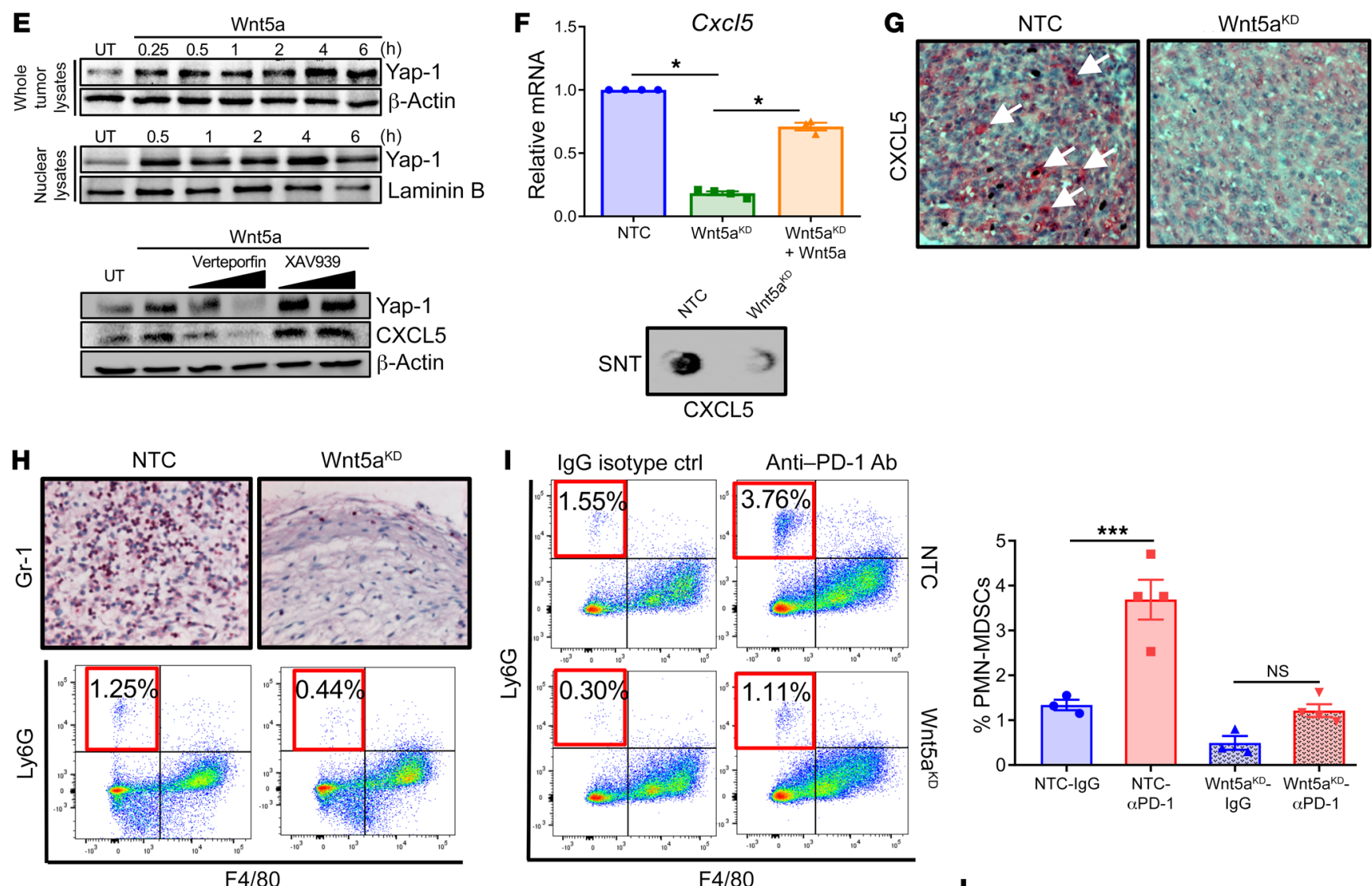
Figure 2. Wnt5a induces CXCR2-dependent chemokine expression in response to anti-PD-1 Ab immunotherapy. (A) TCCA human melanoma database gene expression analysis of $C X C L 5, C X C L 2$, and $C X C R 2$ association with WNT5A. (B) Whole tumor tissue Western blot analysis of Wnt5a, YAP1, CXCL5, and vinculin and $\beta$-actin (used as loading controls). Blot is representative of 3 independent experiments. (C) Plasma CXCL5 ELISA following anti-PD-1 $A b$ therapy versus IgG isotype control therapy in the transgenic BRAF ${ }^{\mathrm{V} 600 \mathrm{E}} \mathrm{PTEN}^{-/-}$melanoma model $(n=6)$. Data are representative of 3 independent experiments. (D) qRT-PCR analysis of $C x C / 1, C x C / 2$, and $C x C / 5$ in the BRAF ${ }^{\mathrm{V} 600 \mathrm{E}} \mathrm{PTEN}^{-1-}$ melanoma cell line following treatment with rWnt5a versus vehicle control $(n=3)$. (E) Western blot analysis of YAP1 expression in total cellular lysates (top) and nuclear lysates (middle) following treatment of BRAF ${ }^{\mathrm{V} 600 \mathrm{E}} \mathrm{PTEN}^{-1-}$ melanoma cells with $\mathrm{rWnt5a}$ at various time points. Bottom blot shows Wnt5a induction of CXCL5 with or without verteporfin (YAP inhibitor) or XAV939 ( $\beta$-catenin inhibitor). Blots shown are representative of 3 independent experiments. UT, untreated or vehicle control. (F) qRT-PCR analysis of $C X C / 5$ in BRAFV600E PTEN $^{-1-}$ NTC and Wnt5a-silenced BRAF ${ }^{\mathrm{V} 600 \mathrm{E}} \mathrm{PTEN}^{-/-}$melanoma cells (BRAF ${ }^{\mathrm{V} 600 \mathrm{E}}$ PTEN $^{-/-}$Wnt5 $5 a^{\mathrm{KD}}$ ). Blot shows secreted CXCL5 in BRAF ${ }^{\mathrm{V} 600 \mathrm{P}} \mathrm{PTEN}^{-1-}$ NTC and BRAF ${ }^{V 600 E}$ PTEN $^{-1-}$ Wnt5 ${ }^{\mathrm{KD}}$ cells $(n=3)$. (C) IHC for CXCL5 (red) in BRAF $^{\mathrm{V} 600 \mathrm{E}} \mathrm{PTEN}^{-/-} \mathrm{NTC}^{-1}$ and BRAF ${ }^{\mathrm{V} 600 \mathrm{E}} \mathrm{PTEN}^{-1-}$ Wnt5 $\mathrm{a}^{\mathrm{KD}}$ tumor cells. Images are representative of 3 tumors. White arrows indicate $C X C L 5^{+}$tumor cells. Original magnification, $\times 20$. (H) IHC for Gr-1 in BRAF ${ }^{\mathrm{V} 600 \mathrm{E}} \mathrm{PTEN}^{-1-}$ NTC and $\mathrm{BRAF}^{\mathrm{V} 600 \mathrm{E}} \mathrm{PTEN}^{-/-}$Wnt5 $\mathrm{a}^{\mathrm{KD}}$ tumor cells. Original magnification, $\times 20$. Plots show PMN-MDSC flow cytometric analysis of BRAFV600E PTEN $^{-1-}$ NTC and BRAF $^{V 600 E}$ PTEN $^{-/-}$Wnt5a ${ }^{\mathrm{KD}}$ tumors $(n=3)$. (I) PMN-MDSC flow cytometric analysis of BRAF ${ }^{\mathrm{V} 600 \mathrm{E}} \mathrm{PTEN}^{-1-} \mathrm{NTC}$ and BRAF ${ }^{\mathrm{V} 600 \mathrm{E}} \mathrm{PTEN}^{-1-} \mathrm{Wnt}^{\mathrm{K}} \mathrm{a}^{\mathrm{KD}}$ tumors following treatment with anti-PD-1 Ab versus IgG isotype control $(n=5)$. (J) Tumor volume change based on anti-PD-1 Ab/lgG control ratios for BRAF $^{\mathrm{V} 600 \mathrm{E}} \mathrm{PTEN}^{-/-} \mathrm{NTC}^{-}$and BRAF ${ }^{\mathrm{V} 600 \mathrm{E}} \mathrm{PTEN}^{-/-} \mathrm{Wnt}^{\mathrm{K}} \mathrm{a}^{\mathrm{KD}}$ tumors $(n=5)$.

$\alpha$, anti. UT, untreated control. Kendall's tau correlation coefficient was calculated for $\mathbf{A} .{ }^{*} P<0.05$ and ${ }^{* * *} P<0.0005$, by Student's $t$ test (C, $\mathbf{D}$, and I) and 1-way ANOVA with Sidak's post hoc multiple comparisons test (F). See also Supplemental Figure 3.

a linear relationship between the number of infiltrating $\mathrm{CD} 8^{+} \mathrm{T}$ cells and the number of PMN-MDSCs per gram of tissue following anti-PD-1 Ab therapy but not in response to IgG isotype control $\mathrm{Ab}$ therapy (Figure 4B). In line with our previous results showing that the elimination of $\mathrm{CD} 8^{+} \mathrm{T}$ cells abolished the increase in tumor CXCL5 expression with anti-PD-1 Ab therapy (Figure 1F), further in vivo experiments showed that $\mathrm{Ab}$-mediated ablation of $\mathrm{CD} 8^{+}$ $\mathrm{T}$ cells also diminished PMN-MDSC recruitment in response to anti-PD-1 Ab therapy (Figure 4C and Supplemental Figure 5C). Together, these data suggest that $\mathrm{CD} 8^{+} \mathrm{T}$ cell activity contributes to the induction of PMN-MDSC recruitment and that this process involves tumor-dependent secretion of HSP70.

Since HSP release by tumors could be interpreted to be due simply to tumor cell death, we inquired whether cytotoxic chemotherapy could induce similar effects. Dacarbazine chemotherapy has historically been used for the management of metastatic melanoma. Although dacarbazine was capable of inducing $\mathrm{BRAF}^{\mathrm{V} 600 \mathrm{E}}$ $\mathrm{PTEN}^{-/-}$melanoma cell death in vitro, this process was not associated with increased HSP7O release (Figure 4D and Supplemental Figure 5B). Consistent with this effect, dacarbazine suppressed $\mathrm{BRAF}^{\mathrm{V} 600 \mathrm{E}} \mathrm{PTEN}^{-/-}$melanoma progression in vivo but did not influence the recruitment of PMN-MDSCs or the numbers of tumor-infiltrating $\mathrm{CD} 8^{+} \mathrm{T}$ cells (Figure 4, $\mathrm{E}$ and $\mathrm{F}$ ).

In light of these findings, we investigated the mechanism regulating tumor $\mathrm{HSP} 70$ secretion in response to $\mathrm{CD} 8^{+} \mathrm{T}$ cell activity. Prior studies have implicated ATP as a stimulator of HSP70 release
(32). Since ATP is also a classic activator of the NLRP3 inflammasome, we hypothesized that NLRP3 played a role in promoting the release of HSP7O using a similar mechanism responsible for its induction of IL-1 $\beta$ and IL-18 secretion (33). To address this question, we conducted in vitro experiments, which showed that a NLRP3 inhibitor could block both ATP stimulation and CD8 ${ }^{+}$ $\mathrm{T}$ cell-mediated induction of HSP70 release from the BRAF ${ }^{\mathrm{V} 600 \mathrm{E}}$ $\mathrm{PTEN}^{-/-}$melanoma cell line (Figures $4, \mathrm{G}$ and $\mathrm{H}$ ). These results support a potential role for the tumor NLRP3 inflammasome in $\mathrm{CD}^{+} \mathrm{T}$ cell-mediated HSP7O release and the observed stimulation of PMN-MDSC recruitment.

$P D-L 1$ triggers NLRP3 activation and downstream activation of the HSP70/Wnt5a signaling axis in tumors. In view of the relationship between infiltrating $\mathrm{T}$ cells and the induction of PD-L1 in the tumor microenvironment via IFN signaling, we further conjectured that release of HSPs from tumors and stimulation of the NLRP3/ HSP70 signaling axis are dependent on PD-L1 upregulation (15). To test this hypothesis, we coincubated $\mathrm{BRAF}^{\mathrm{V} 600 \mathrm{E}} \mathrm{PTEN}^{-/-}$melanoma cells with IFN- $\gamma$ in the absence and presence of an agonistic anti-PD-L1 Ab and monitored for HSP70 release as well as for evidence of caspase- 1 cleavage as a surrogate for NLRP3 activation. This study showed that anti-PD-L1 Ab plus IFN- $\gamma$ treatment of $\mathrm{BRAF}^{\mathrm{V} 600 \mathrm{E}} \mathrm{PTEN}^{-/-}$melanoma cells indeed induced activation of the NLRP3 inflammasome, concurrent release of HSP70, and upregulation of Wnt5a (Figure 5A). This effect was also observed in the LLC cell line, indicating that this phenomenon is not restricted to the $\mathrm{BRAF}^{\mathrm{V} 600 \mathrm{E}} \mathrm{PTEN}^{-/ /}$melanoma model (Supplemental Figure 6A). To further confirm the sequence of this signaling pathway, we genetically silenced HSP70 using a CRISPR/Cas9 approach in the $\mathrm{BRAF}^{\mathrm{V} 600 \mathrm{E}} \mathrm{PTEN}^{-/-}$melanoma cell line and stimulated the upstream NLRP3 inflammasome in these BRAFV $^{\mathrm{V}}{ }^{\circ 0 E} \mathrm{PTEN}^{-/-} \mathrm{HSP}^{-/ /}$cells along with their nontarget control (NTC) cell line. These experiments showed that HSP70 ablation eliminated the ability of the NLRP3 inflammasome to stimulate the upregulation of Wnt5a in both an autocrine and paracrine manner (Supplemental Figure 6B). Further experiments demonstrated that tumor cell PD-L1 cross-linking induced NLRP3 binding to the apoptosis-associated speck-like protein containing a caspase recruitment domain (ASC) adaptor protein, while also promoting ASC polymerization, both of which were necessary to generate the inflammasome macromolecular complex (Figure 5, $\mathrm{B}$ and $\mathrm{C})$. We then verified that $\mathrm{PD}-1$ blockade promotes $\mathrm{CD} 8^{+} \mathrm{T}$ cell induction of NLRP3 activation and tumor HSP70 release in an IFN- $\gamma$-dependent manner in further OT-1 $\mathrm{CD}^{+} \mathrm{T}$ cell: $\mathrm{BRAF}^{\mathrm{V} 600 \mathrm{E}}$ OVA melanoma coculture experiments (Figure 5D). Notably, this induction of NLRP3 activation by $\mathrm{CD} 8^{+} \mathrm{T}$ cells was found to be antigen specific, as $\mathrm{CD} 8^{+} \mathrm{T}$ cells that recognize an irrelevant control peptide do not induce caspase-1 activation (Supplemental Figure 6C). Furthermore, this phenomenon required $\mathrm{T}$ celltumor cell contact or close proximity, as Transwell assays failed to induce caspase-1 cleavage and HSP70 release, consistent with an important role for physical PD-1-PD-L1 interactions (Supplemental Figure 6D). Importantly, both the pharmacologic inhibition and genetic silencing of Nlrp3 effectively suppressed HSP7O release and subsequent Wnt5a upregulation in response to antiPD-1 Ab activation of tumor antigen-specific $\mathrm{CD} 8^{+} \mathrm{T}$ cells (Figure $5, \mathrm{E}$ and $\mathrm{F}$ ). We observed a similar effect with genetic silencing of 
A

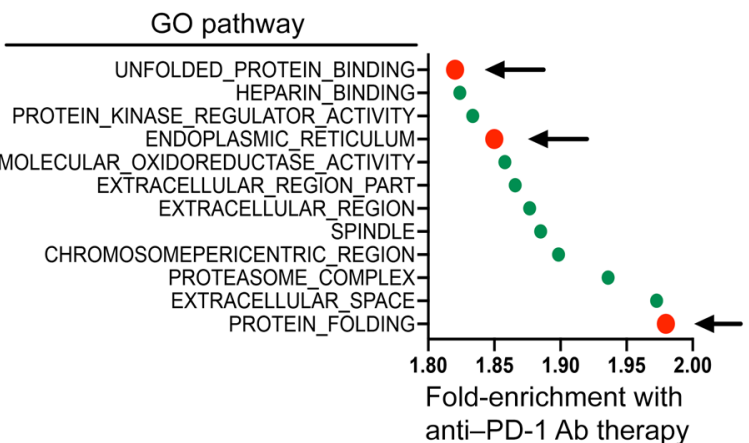

B

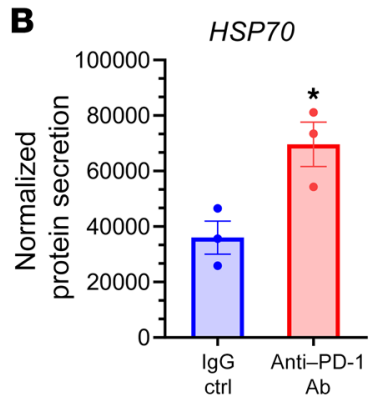

C

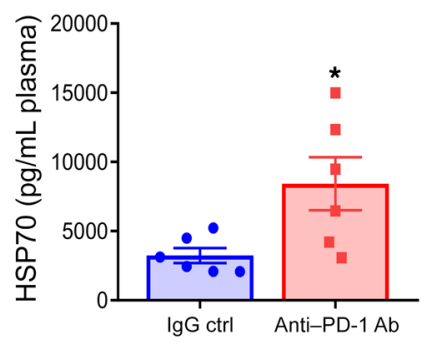

D

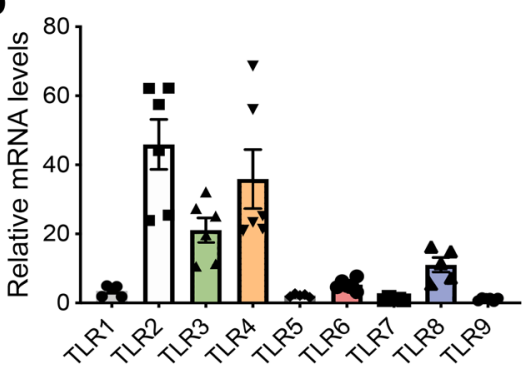

H

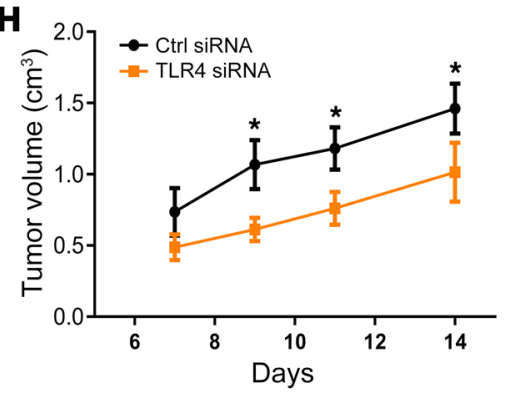

I

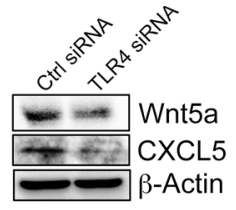

E

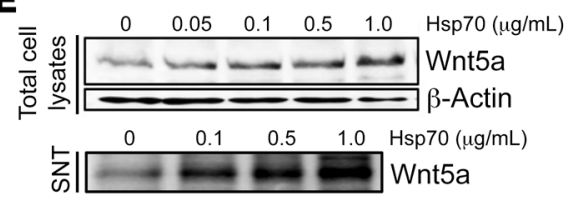

$\mathbf{F}$

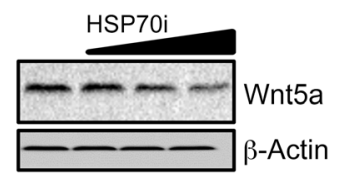

J
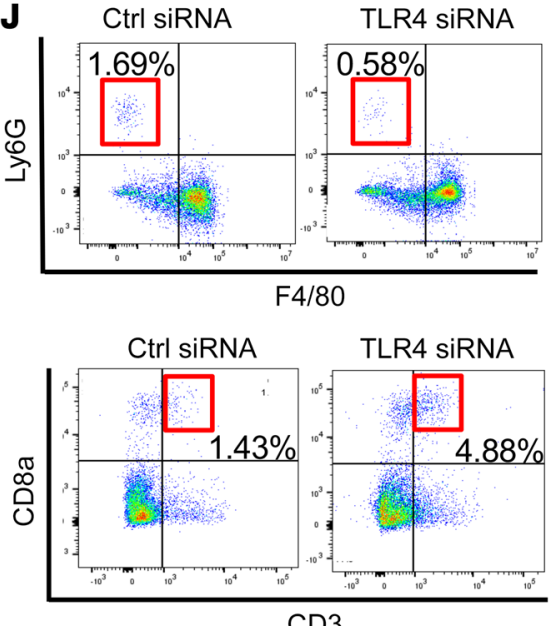
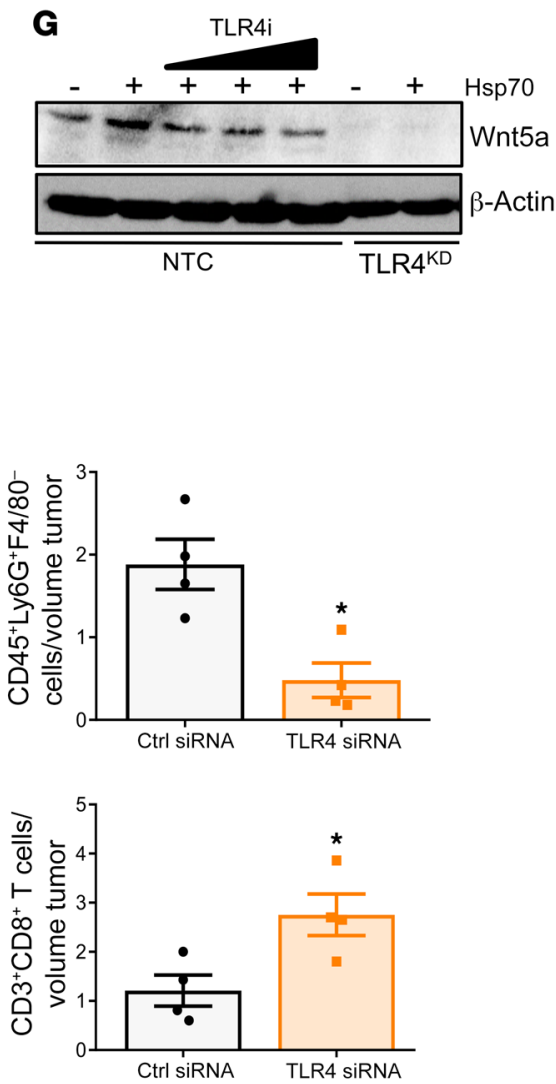

Figure 3. HSP70-TLR4 induces Wnt5a expression in response to anti-PD-1 Ab immunotherapy. (A) RNA-Seq GSEA showing top 12 pathways enriched in autochthonous BRAF ${ }^{\mathrm{V} 600 \mathrm{E}} \mathrm{PTEN}^{-1-}$ melanomas following escape from anti-PD-1 Ab therapy. Arrows indicate pathways associated with cellular stress ( $n=$ 3/group). (B) SILAC-AHA LC-MS/MS secretome analysis of resected autochthonous BRAF ${ }^{\mathrm{V} 600 \mathrm{E}} \mathrm{PTEN}^{-1-}$ melanoma tissues following anti-PD-1 Ab therapy versus IgG isotype control. Secreted protein levels were normalized to the number of cells ( $n=3 /$ group). (C) Plasma HSP70 ELISA analysis following anti$\mathrm{PD}-1$ versus IgG isotype control treatment of autochthonous BRAF ${ }^{\mathrm{V} 600 \mathrm{E}} \mathrm{PTEN}^{-1-}$ melanoma-bearing mice $(n=6)$. (D) qRT-PCR analysis of TLR expression in $\mathrm{BRAF}^{\mathrm{V} 600 \mathrm{E}} \mathrm{PTEN}^{-/-}$melanoma cells. Data were normalized to Tlrg expression levels $(n=3)$. (E) Treatment of BRAF ${ }^{\mathrm{V} 600 \mathrm{E}} \mathrm{PTEN}^{-/-}$melanoma cells with titrated concentrations of recombinant HSP70 ( $r$ HSP70) followed by Wnt5a Western blot analysis of total cell lysates and supernatant (SNT). Blots are representative of 2 independent experiments. (F) Treatment of BRAF ${ }^{\mathrm{V} 600 \mathrm{E}} \mathrm{PTEN}^{-1-}$ melanoma cells with titrated concentrations of the HSP70 inhibitor VER155008 (HSP7Oi). Blots are representative of 2 independent experiments. (G) Treatment of BRAF ${ }^{\mathrm{V} 600 \mathrm{P}}$ PTEN ${ }^{-1-}$ NTC cells with rHSP70 with or without the TLR4 inhibitor CLI-095 (TLR4i) and treatment of TIr4-silenced BRAF ${ }^{\mathrm{V} 600 \mathrm{E}} \mathrm{PTEN}^{-1-}$ melanoma cells (TLR4 ${ }^{\mathrm{KD}}$ ) with HSP70 followed by Western blotting for Wnt5a. Blots are representative of 3 independent experiments. (H) BRAFV00E PTEN ${ }^{-1-}$ melanoma growth curve following treatment with TLR4 siRNA versus control siRNA $(n=5)$. (I) Whole-tissue Western blot analysis of Wnt5a, CXCL5, and $\beta$-actin in TLR4 siRNA-treated and control siRNA-treated BRAFv600E $\mathrm{PTEN}^{-1-}$ melanomas. Data are representative of 2 independent experiments. (J) Top: PMN-MDSC flow cytometric analysis of TLR4 siRNA- and control siRNA-treated BRAF ${ }^{\mathrm{V} 600 \mathrm{E}} \mathrm{PTEN}^{-1-}$ melanomas $(n=4)$. Bottom: CD8 ${ }^{+} \mathrm{T}$ cell flow cytometric analysis of TLR4 siRNA- and control siRNA-treated BRAF ${ }^{\mathrm{V} 600 \mathrm{E}}$ PTEN $^{-1-}$ melanomas $(n=4) .{ }^{*} P<0.05$, by Student's $t$ test for comparison of treatment groups. See also Supplemental Figure 4.

Pdl1 in the BRAF ${ }^{\mathrm{V} 600 \mathrm{E}} \mathrm{PTEN}^{-/-}$melanoma cell line, which resulted in elimination of ASC polymerization, caspase- 1 activation, HSP70 secretion, and Wnt5a upregulation (Figure 5, C and F, and Supplemental Figure 6E).
On the basis of these data, we examined the mechanism mediating PD-L1-dependent NLRP3 activation. dsRNA-dependent protein kinase $\mathrm{R}$ (PKR) is a known activator of all inflammasome proteins, including NLRP3, and has been shown to regulate cer- 

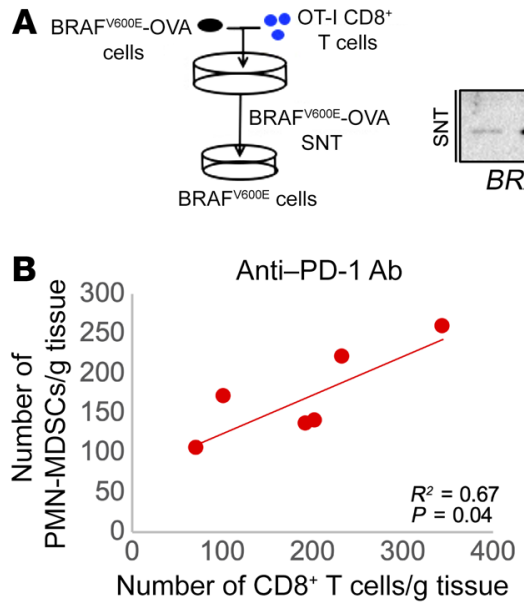
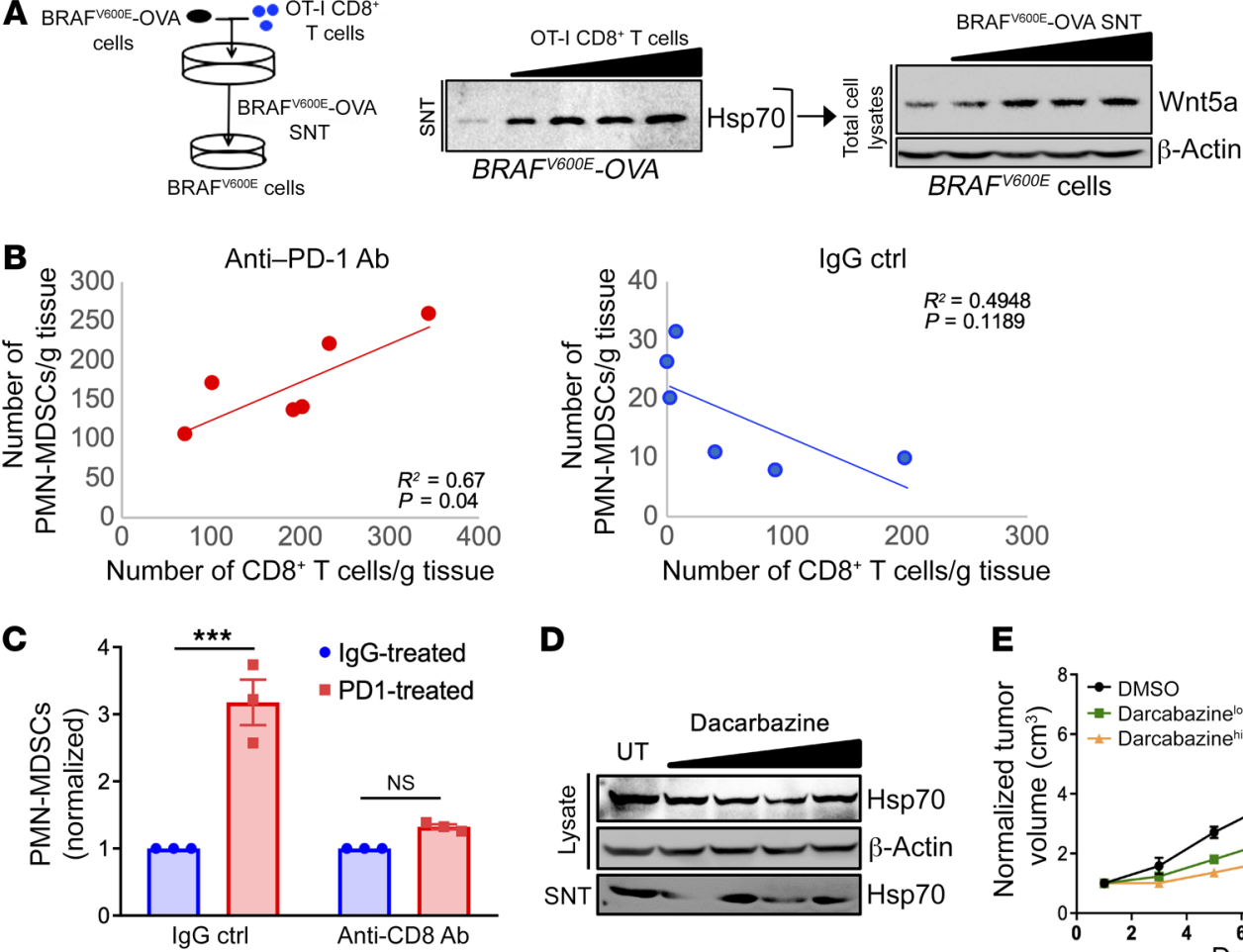

$\lg \mathrm{ctrl}$

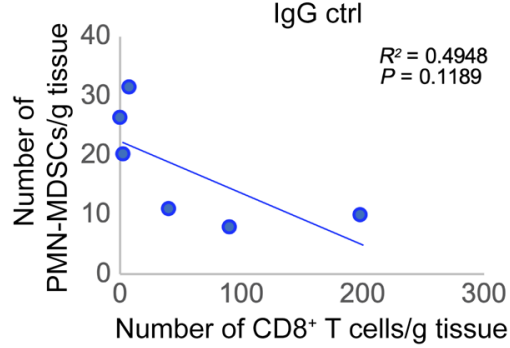

D

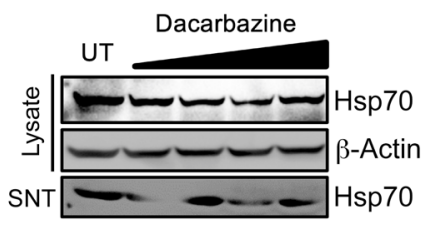

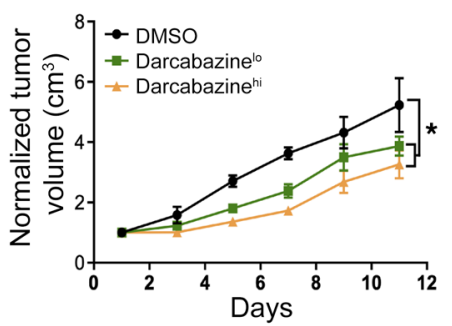
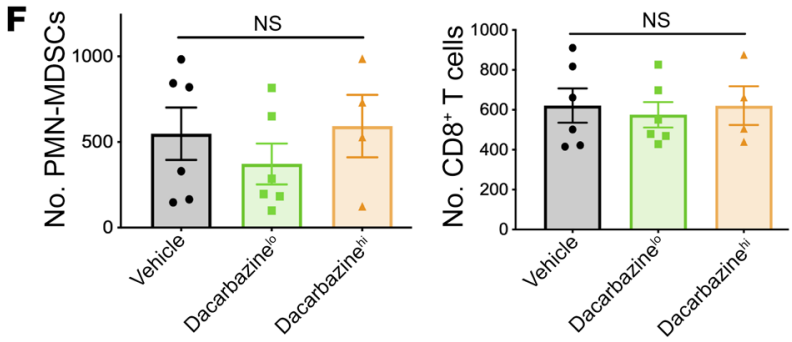

G
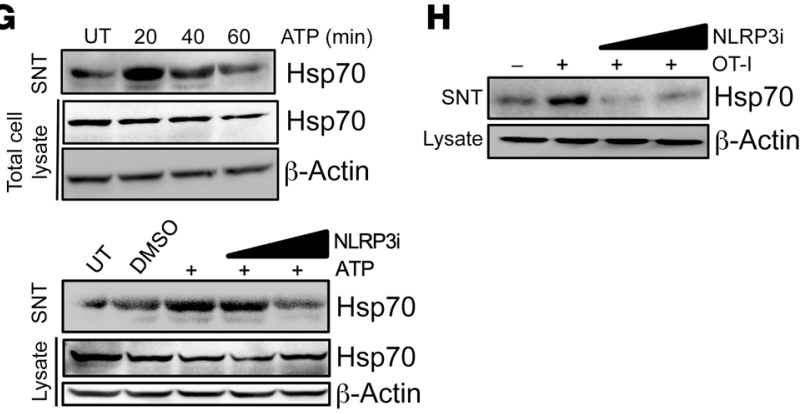

Figure 4. CD8+ T cells induce tumor HSP70 release in a NLRP3-dependent manner in response to anti-PD-1 Ab immunotherapy. (A) Schema illustrating coculture of OT-I CD8 ${ }^{+}$T cells with OVA-expressing BRAF ${ }^{\mathrm{V} 600 \mathrm{E}} \mathrm{PTEN}^{-1-}$ melanoma cells followed by HSP70 Western blot analysis of isolated supernatant. Harvested supernatant was coincubated at increasing concentrations with WT BRAF ${ }^{\mathrm{V} 600 \mathrm{E}} \mathrm{PTEN}^{-/-}$melanoma cells followed by Wnt5a Western blot analysis. Blots are representative of 2 independent experiments. (B) Flow cytometric analysis of PMN-MDSCs and CD8 ${ }^{+} \mathrm{T}$ cells from resected autochthonous BRAF $^{\mathrm{V} 600 E} \mathrm{PTEN}^{-/-}$melanoma tissues following anti-PD-1 Ab or lgG isotype control therapy. Results are expressed per gram of tumor tissue $(n=6)$. (C) Flow cytometric analysis of tumor-infiltrating PMN-MDSCs from autochthonous BRAFV60E PTEN ${ }^{-1-}$ melanomas following anti-PD-1 Ab versus IgC isotype control therapy with or without anti-CD8 Ab. Data were normalized to lgG control-treated tumors $(n=3)$. (D) HSP70 and $\beta$-actin Western blot analysis following treatment of BRAF ${ }^{\mathrm{V} 600 \mathrm{E}} \mathrm{PTEN}^{-/-}$melanoma cells with increasing concentrations of dacarbazine. Blots are representative of 3 independent experiments. (E) Tumor growth curve of syngeneic BRAF ${ }^{\mathrm{V} 600} \mathrm{PTEN}^{-/-}$melanomas following vehicle control or low-dose (lo) (50 mg/kg i.p. q.o.d.) or high-dose (hi) (75 mg/kg i.p. q.o.d.) dacarbazine therapy $(n=5)$. (F) Flow cytometric analysis of PMN-MDSCs from BRAF ${ }^{\mathrm{V} 600 \mathrm{E}} \mathrm{PTEN}^{-1-}$ melanomas following vehicle control or dacarbazine therapy $(n=5)$. Flow cytometric analysis of CD8 ${ }^{+}$T cells from BRAF ${ }^{\mathrm{V} 600 \mathrm{E}} \mathrm{PTEN}^{-/-}$melanomas following vehicle control or dacarbazine therapy $(n=5)$. (G) HSP70 Western blot analysis of supernatant and tumor cell lysates following ATP stimulation of BRAFV600E PTEN ${ }^{-1-}$ melanoma cells at different time points, with or without treatment with the NLRP3 inhibitor (NLRP3i) MCC950. Blots are representative of 3 independent experiments. (H) HSP70 Western blot following coincubation of OT-1 CD8 ${ }^{+}$T cells and OVA-expressing BRAF ${ }^{\mathrm{V} 600 \mathrm{P}} \mathrm{PTEN}^{-1-}$ melanoma cells with or without increasing concentrations of NLRP3 inhibitor. Blots are representative of 3 independent experiments. Spearman's correlation calculation was performed in $\mathbf{B}$. ${ }^{*} P<0.05$ and ${ }^{* * *} P<$ 0.0005 , by Student's $t$ test ( C), 1-way ANOVA with Sidak's post hoc multiple comparisons test (E and F). See also Supplemental Figure 5.

tain inflammatory pathways (34). Indeed, PKR inhibition suppressed caspase-1 cleavage in the BRAF ${ }^{\mathrm{V} 600 \mathrm{E}} \mathrm{PTEN}^{-/-}$melanoma cell line in response to anti-PD-L1 Ab/IFN- $\gamma$ stimulation (Supplemental Figure 6F). Consistent with these studies, further treatment of $\mathrm{BRAF}^{\mathrm{V} 600 \mathrm{E}} \mathrm{PTEN}^{-/-}$melanoma cells with anti-PD-L1 Ab and IFN- $\gamma$ induced PKR-NLRP3 binding, whereas genetic silencing of Pdl1 also suppressed PKR phosphorylation (Figure 5, G and
H). A recent report has shown that tumor-intrinsic PD-L1 signaling inhibits STAT3 activation (23). Previous work has also shown that cytosolic STAT3 inhibits PKR kinase activity and suppresses activation of the NLRP3 inflammasome $(35,36)$. We therefore hypothesized that tumor PD-L1 promotes PKR-NLRP3 activation by suppressing STAT3 levels. Indeed, we found that Pdl1 silencing upregulated total STAT3 levels concurrently with diminished PKR 

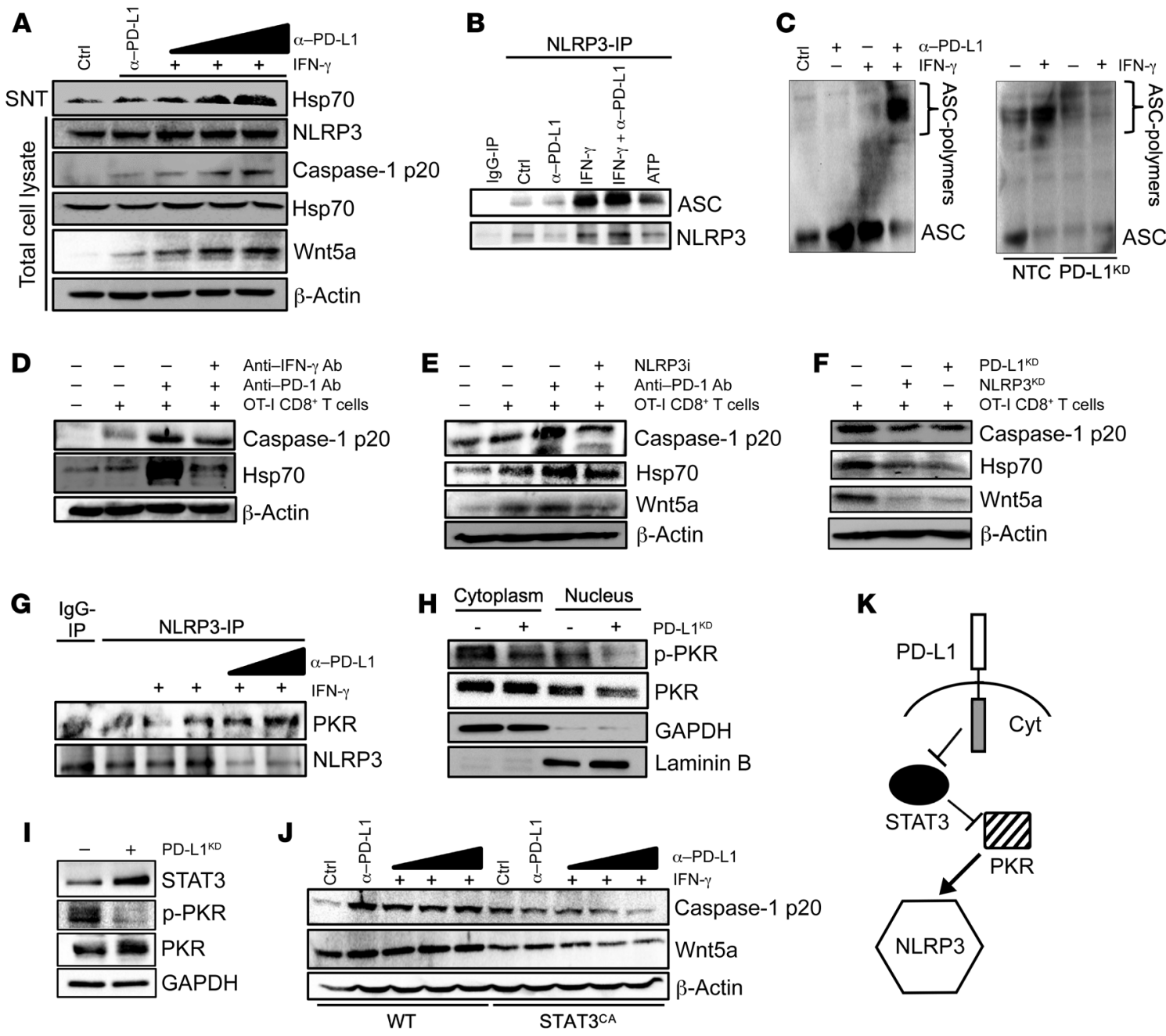

Figure 5. CD8+ T cells trigger a PD-L1/NLRP3 signaling pathway to drive PMN-MDSC recruitment to the tumor. (A) Western blots for HSP70 supernatant, caspase-1 p20, and Wnt5a in BRAF ${ }^{V 600 E}$ PTEN $^{-1-}$ melanoma cells treated with anti-PD-L1 Ab with or without IFN- $\gamma$. (B) Immunoprecipitation (IP) of NLRP3 after treatment of BRAF ${ }^{\mathrm{V} 600 \mathrm{E}} \mathrm{PTEN}^{-1-}$ melanoma cells with IFN- $\gamma$, anti-PD-L1, or both followed by Western blotting for ASC and NLRP3. IgG-IP, IP control; ATP, positive control. (C) Left: ASC polymerization assay following treatment of BRAF ${ }^{\mathrm{V} 600 \mathrm{P}} \mathrm{PTEN}^{-/-}$melanoma cells with IFN- $\gamma$, anti-PD-L1, or both. Right: ASC polymerization assay following treatment of PdI7-silenced and NTC BRAFV600E PTEN ${ }^{-1-}$ melanoma cells with IFN- $\gamma$. (D) Coculture of OT-I CD8 ${ }^{+}$T cells with OVA-expressing BRAF ${ }^{\mathrm{V} 600 \mathrm{E}} \mathrm{PTEN}^{-/-}$melanoma cells, with or without anti-PD-1 Ab alone or anti-PD-1 Ab plus anti-IFN- $\gamma$-blocking Ab, was followed by Western blotting for HSP70 and caspase-1 p2O. (E) Coculture of OT-I CD8 ${ }^{+}$T cells with BRAFV600E PTEN $^{-1-}$ OVA melanoma cells, with or without anti-PD-1 Ab alone or anti-PD-1 Ab plus NLRP3 inhibitor, was followed by Western blots for caspase-1 p20, HSP70, and Wnt5a. (F) Western blots for caspase-1 p20, HSP70, and Wnt5a Western blots in BRAF ${ }^{\mathrm{V} 600 \mathrm{E}} \mathrm{PTEN}^{-1-}$ OVA melanoma cells following coculture with OT-I CD8 ${ }^{+}$T cells after genetic silencing of either NIrp3 (NRLP3 ${ }^{\mathrm{KD}}$ ) or PdI1 (PD-L1 ${ }^{\mathrm{KD}}$ ). (C) IP of NLRP3 after treatment of BRAFV600E PTEN $^{-1-}$ melanoma cells with IFN- $\gamma$, anti-PD-L1, or both, followed by Western blotting for PKR and NLRP3. (H) Western blots for $\mathrm{p}$-PKR and total PKR in control and Pdl1-silenced BRAF ${ }^{\mathrm{V} 600 \mathrm{E}} \mathrm{PTEN}^{-/-}$melanoma cells. GAPDH was used as a cytoplasmic loading control and laminin B as a nuclear loading control. (I) Western blotting for STAT3, p-PKR, and total PKR in control and Pdl1silenced BRAF ${ }^{\mathrm{V} 600 \mathrm{E}} \mathrm{PTEN}^{-/-}$melanoma cells. (J) Western blots for caspase-1 p20 and Wnt5a in WT and STAT3 ${ }^{\mathrm{CA}}$-expressing BRAF ${ }^{\mathrm{V} 600 \mathrm{E}} \mathrm{PTEN}^{-/-}$melanoma cells following treatment with IFN- $\gamma$, anti-PD-L1, or both. (K) Schematic diagram depicting the PD-L1/STAT3/PKR/NLRP3 signaling axis in tumor cells. cyt, cytoplasm. All Western blots are representative of 2-3 independent experiments. See also Supplemental Figure 6.

phosphorylation (Figure 5I). Consistent with a suppressive role for STAT3 in this pathway, we found that constitutive activation of STAT3 (STAT3 ${ }^{\mathrm{CA}}$ ) suppressed NLRP3 activation, as indicated by the diminished caspase- 1 cleavage and Wnt5a expression levels (Figure 5J). These data indicate that PD-L1 triggers PKRdependent activation of the NLRP3 inflammasome in tumors by repressing STAT3 (Figure 5K). In summary, we have elucidated a mechanistic link between PD-L1 and the tumor-intrinsic NLRP3 inflammasome and showed that this pathway drives adaptive immune evasion by promoting the recruitment of PMN-MDSCs.
Genetic and pharmacologic inhibition of NLRP3 blocks PMN$M D S C$ recruitment and enhances the efficacy of anti-PD-1 Ab immunotherapy. Given the central role of the NLRP3 inflammasome in mediating PMN-MDSC recruitment to the tumor bed in response to anti-PD-1 Ab therapy, we hypothesized that inhibiting NLRP3 activation would promote antitumor immunity and suppress tumor progression. Using a shRNA-expressing lentiviral vector, we silenced Nlrp3 in $\mathrm{BRAF}^{\mathrm{V} 600 \mathrm{E}} \mathrm{PTEN}^{-/-}$melanoma cells and transplanted this cell line into syngeneic hosts (Supplemental Figure 7A). After 20 days of tumor growth, Nlrp3-silenced BRAF ${ }^{\mathrm{V} 600 \mathrm{E}}$ 
$\mathrm{PTEN}^{-/-}$melanomas were associated with reduced plasma HSP70 levels, decreased levels of CXCR2 ligand expression, and diminished PMN-MDSC infiltration relative to control BRAF ${ }^{\mathrm{V} 600 \mathrm{E}}$ $\mathrm{PTEN}^{-/-}$melanomas (Figure 6, A-C). Although Nlrp3 silencing did not influence tumor cell proliferation in vitro, it increased the levels of tumor-infiltrating $\mathrm{CD}^{+} \mathrm{T}$ cells and suppressed the growth of $\mathrm{BRAF}^{\mathrm{V} 600 \mathrm{E}} \mathrm{PTEN}^{-/-}$melanomas in vivo. (Figure 6, C and D, and Supplemental Figure 7, B and C). NLRP3-dependent regulation of the antitumor immune response was further confirmed by additional in vivo tumor experiments, in which $\mathrm{CD} 8^{+} \mathrm{T}$ cell ablation reversed tumor growth suppression in Nlrp3-silenced BRAF ${ }^{\mathrm{V} 600 \mathrm{E}}$ $\mathrm{PTEN}^{-/-}$melanomas (Supplemental Figure 7D).

We performed additional studies to determine whether systemic pharmacological inhibition of NLRP3 could also suppress tumor growth and augment anti-PD-1 Ab immunotherapy in the BRAF ${ }^{\mathrm{V} 600 \mathrm{E}}$ $\mathrm{PTEN}^{-/-}$melanoma model. Using the NLRP3 inhibitor MCC950, we also found that systemic NLRP3 inhibition diminished PMN-MDSC recruitment in response to anti-PD-1 Ab therapy, enhanced levels of tumor-infiltrating $\mathrm{CD} 8^{+} \mathrm{T}$ cells, and suppressed tumor progression in vivo beyond what was observed with anti-PD-1 Ab monotherapy (Figure 6, E and F) (37). Whole-tissue Western blot analysis, plasma ELISAs, and tumor qRT-PCR studies further showed that these effects correlated with suppressed caspase- 1 cleavage, Wnt5a expression, Cxcl5 levels, and HSP70 release and enhanced expression of the cytolytic T cell marker Gzmb (Figure 6, G and H, and Supplemental Figure 7E). Notably, neither pharmacologic inhibition of NLRP3 nor genetic silencing of Nlrp3 had any impact on tumor cell proliferation in vitro (Supplemental Figure 7C). Altogether, these data are consistent with our previous studies supporting the critical role of the NLRP3 inflammasome in driving PMN-MDSC recruitment as an adaptive response to $\mathrm{CD} 8^{+} \mathrm{T}$ cell activation and suggest that NLRP3 is a viable pharmacologic target for enhancing the efficacy of anti-PD-1 Ab therapy.

$P M N-M D S C$ recruitment as a mechanism of adaptive resistance to anti-PD-1 Ab therapy in human melanoma. In order to determine whether PD-L1 can trigger NLRP3 activation and HSP70 release in human melanoma, we performed in vitro experiments using the WM266 human melanoma cell line. Similar to our previous observations, tumor PD-L1 cross-linking following IFN- $\gamma$ stimulation induced caspase-1 cleavage and HSP70 release (Figure 7A). Further studies using the WM266 human melanoma cell line also showed that HSP70 induced Wnt5a upregulation in a TLR4-dependent manner and that pharmacologic NLRP3 inhibition suppressed ATP-mediated HSP70 release (Figure 7, B and $\mathrm{C}$ ). These data were consistent with a significant correlation between expression of the myeloid markers ITGAM and CD33 as well as NLRP3 and the cytolytic T cell markers CD8A, GZMB, and PRF1 in human metastatic melanoma specimens, based on RNA expression data in TCGA-SKCM database (Figure 7D). These data are consistent with our previous observations in the autochthonous murine melanoma model and suggest that $\mathrm{CD}^{+} \mathrm{T}$ cell activation results in tumor release of HSP70, Wnt5a upregulation, and recruitment of infiltrating myeloid cells (Figure 1A). To further study this mechanism, we harvested human melanoma tissue specimens at baseline and during disease progression following nivolumab anti-PD-1 Ab immunotherapy and performed RNASeq differential whole transcriptomic sequencing analysis. Con- sistent with our preclinical studies, these studies revealed elevated expression of several myeloid markers including CXCL1, CD33, ITGAM, CXCR2, S100A8, and S100A9 following disease progression through checkpoint inhibitor immunotherapy (Figure 7E).

Our previous work showed that tumors release HSP7O in response to $\mathrm{CD}^{+} \mathrm{T}$ cell responses and that this increase in HSP70 levels can be measured in the plasma of mice undergoing antiPD-1 Ab immunotherapy (Figure 3C and Supplemental Figure 7E). On the basis of these data, we conducted a plasma-based ELISA to quantitate HSP70 levels at baseline and at week 12 of anti-PD-1 Ab immunotherapy in patients with advanced melanoma undergoing anti-PD-1 Ab immunotherapy. This study revealed that HSP7O levels increased in patients who progressed on anti-PD-1 Ab therapy, but these changes were seldom observed in responders (Figure 7F). Indeed, mean changes in plasma HSP70 levels with anti-PD-1 $\mathrm{Ab}$ therapy were significantly greater in nonresponding melanoma patients relative to responders (Figure 7G). This relationship was also observed after normalizing quantitative HSP70 levels to tumor burden based on CT imaging, indicating that this finding was not strictly due to disease progression. These observations suggest that the process leading to HSP70 release correlates with resistance to checkpoint inhibitor therapy, providing further support for the key role of the NLRP3 inflammasome in the evolution of adaptive resistance to anti-PD-1 Ab immunotherapy.

\section{Discussion}

Using several preclinical models in addition to clinical specimens, we have characterized a PD-L1-dependent, tumor-intrinsic signaling pathway that directly links $\mathrm{CD}^{+} \mathrm{T}$ cell activity with the recruitment of PMN-MDSCs to the tumor bed in response to anti-PD-1 Ab immunotherapy. We propose that this signaling cascade constitutes an adaptive resistance pathway that, when blocked, can enhance the efficacy of anti-PD-1 Ab checkpoint inhibitor immunotherapy. After recognizing a consistent increase in PMN-MDSCs in tumor tissues following anti-PD-1 Ab escape in several tumor models, we traced the underlying mechanism of this phenomenon to a process that involves PD-L1-dependent activation of the NLRP3 inflammasome in tumor tissues. Together, these data highlight several potential pharmacologic targets that may be capable of enhancing immunotherapy efficacy, as well as promising candidate biomarkers that may contribute to improved patient selection and management of patients with cancer undergoing immunotherapy.

Prior studies demonstrating that noncanonical Wnt ligands can induce the activation of YAP-dependent signaling pathways, coupled with data showing that YAP signaling can regulate the expression of several CXCR2-dependent chemokines, prompted us to explore a role for Wnt5a in mediating the observed influx of PMN-MDSCs in response to checkpoint inhibitor therapy (27, 28). These studies demonstrated that tumor Wnt5a expression was induced in response to anti-PD-1 Ab therapy and that the genetic silencing of tumor Wnt5a expression eliminated adaptive recruitment of PMN-MDSCs in response to checkpoint inhibitor immunotherapy. A recent RNA-Seq-based study found Wnt5a to be significantly upregulated in PD-1-refractory melanoma tissues, whereas other studies identified Wnt5a as a marker of dedifferentiation, disease aggressiveness, and therapeutic resistance (17, 

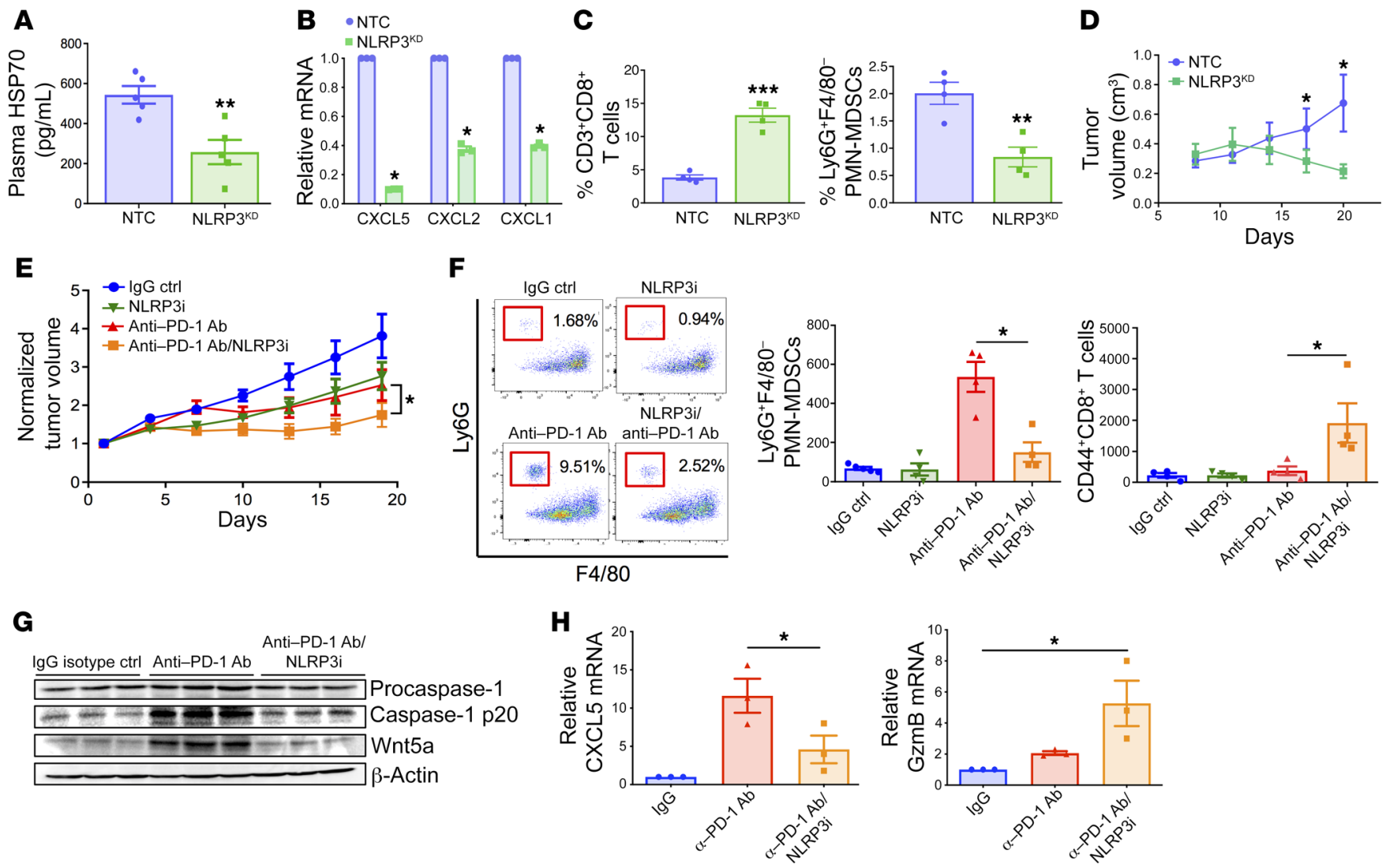

Figure 6. Genetic and pharmacologic inhibition of NLRP3 suppresses PMN-MDSC recruitment and enhances the efficacy of anti-PD-1 Ab immunotherapy. (A) Plasma HSP70 ELISA analysis following the growth of BRAF ${ }^{\mathrm{V} 600 \mathrm{E}} \mathrm{PTEN}^{-1-}$ NTC or Nlrp3-silenced BRAFV600E PTEN $\mathrm{N}^{-1-}$ melanomas $(n=5)$. (B) qRT-PCR analysis of CXCR2-dependent chemokine expression in BRAF ${ }^{V 600 E}$ PTEN $^{-1-}$ NTC and BRAF ${ }^{\mathrm{V} 600 E} \mathrm{PTEN}^{-/-} \mathrm{NLRP}^{\mathrm{KD}}$ melanomas $(n=3)$. (C) Flow cytometric analysis of $\mathrm{CD}^{+} \mathrm{T}^{\mathrm{T}}$ cells in resected BRAF ${ }^{\mathrm{V} 600 \mathrm{E}} \mathrm{PTEN}^{-1-} \mathrm{NTC}^{-}$and BRAF ${ }^{\mathrm{V} 600 \mathrm{E}} \mathrm{PTEN}^{-1-} \mathrm{NLRP3}^{\mathrm{KD}}$ melanomas $(n=5)$. Flow cytometric analysis of PMN-MDSCs in resected

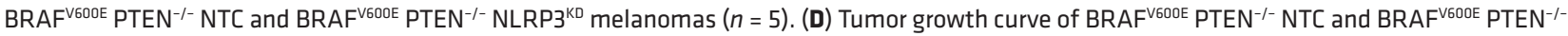

$\mathrm{NLRP}^{\mathrm{KD}}$ melanomas $(n=5)$. (E) Treatment of syngeneic BRAF ${ }^{\mathrm{V} 600 \mathrm{E}} \mathrm{PTEN}^{-1-}$ melanomas with IgG isotype control Ab (200 $\mu$ g i.p. every 3 days), NLRP3 inhibitor (10 $\mu$ g MCC950 i.p. every 3 days), anti-PD-1 Ab (200 $\mu$ g i.p. every 3 days), or NLRP3 inhibitor and anti-PD-1 Ab combination therapy ( $n=8)$. (F) Representative flow cytometric dot plots of PMN-MDSCs and CD8 ${ }^{+} \mathrm{T}$ cells in resected BRAF ${ }^{\mathrm{V} 600 \mathrm{E}} \mathrm{PTEN}^{-/-}$melanomas following treatment with IgC isotype control Ab, NLRP3 inhibitor, anti-PD-1 Ab, or NLRP3 inhibitor and anti-PD-1 Ab combination therapy. Graphs show flow cytometric analysis of tumor-infiltrating PMN-MDSCs and CD44+CD8+ T cells. (C) Whole tumor tissue Western blot analysis for pro-caspase-1, caspase-1 p20, and Wnt5a following in vivo treatment with IgG isotype control, anti-PD-1 Ab, or combined anti-PD-1 Ab and NLRP3 inhibitor. Blots are representative of 2 independent experiments. (H) qRT-PCR analysis of $C x C / 5$ and granzyme B $(\mathrm{Gzmb})$ expression in resected BRAF ${ }^{\mathrm{V}}{ }^{\circ 00 E} \mathrm{PTEN}^{-1-}$ melanoma tissues $(n=5)$. ${ }^{*} P<0.05$, ${ }^{* *} P<0.005$, and ${ }^{* * *} P$ $<0.0005$, by Student's $t$ test $(\mathbf{A}-\mathbf{D})$ and 1-way ANOVA with Sidak's post hoc multiple comparisons test (E, F, and $\mathbf{H})$. See also Supplemental Figure 7 .

$18,38,39)$. However, a mechanistic description of how Wnt5a promotes immune tolerance and immunotherapy resistance remains incomplete. The present study indicates that autocrine Wnt5a signaling could promote PMN-MDSC recruitment to the tumor bed, which is consistent with our prior studies demonstrating a role for paracrine Wnt5a signaling in the induction of DC indoleamine 2,3-dioxgenase expression and enzymatic activity and the subsequent promotion of DC-mediated Treg differentiation $(19,40)$. Overall, these data provide further support for the idea that Wnt ligand-mediated signaling in the tumor microenvironment promotes immune evasion and that targeting Wnt ligand signaling is a promising option for modulating tumor immunity and responses to checkpoint inhibitor immunotherapy.

When evaluating the underlying mechanism driving Wnt5a upregulation in response to anti-PD-1 Ab therapy in these models, we noted evidence of a considerable level of HSP release by tumor cells, based on LC-MS/MS secretome analysis. Tumor release of HSP70 was further confirmed by Western blot analysis and ELISA in response to cytolytic $\mathrm{CD} 8^{+} \mathrm{T}$ cell activity in vitro and in vivo, respectively. This finding was of interest since previous studies have demonstrated that HSP70 can promote cancer progression and that TLRs both mediate HSP70 signaling and have been implicated in the regulation of Wnt5a expression in macrophages $(20,29,41,42)$. We subsequently demonstrated that HSP70 is capable of stimulating Wnt5a expression in various cell lines and that genetic silencing and pharmacologic inhibition of tumor TLR4 blocked tumor Wnt5a and CXCL5 expression, while also inhibiting PMN-MDSC recruitment to developing tumors. Notably, the release of HSP7O was not found to be due simply to a cell death-dependent mechanism, as dacarbazine chemotherapy readily induced tumor apoptosis in the $\mathrm{BRAF}^{\mathrm{V} 600 \mathrm{E}}$ melanoma model but did not induce significant levels of HSP70 release. Although HSP70 has been identified within tumor-derived exosomes, prior studies have also suggested that soluble HSP70 may be released 
A

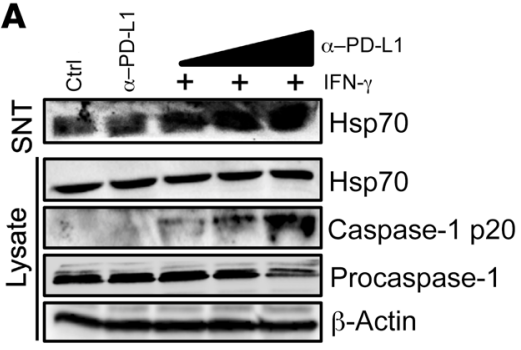

D
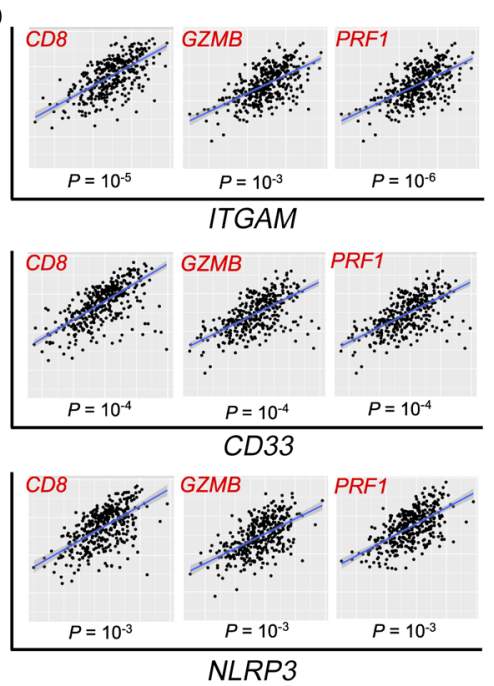

B

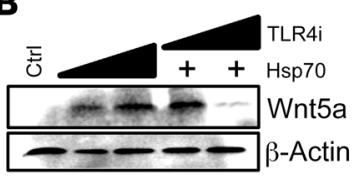

C

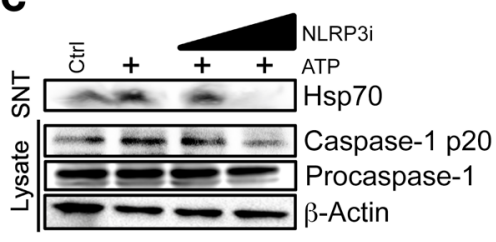

E

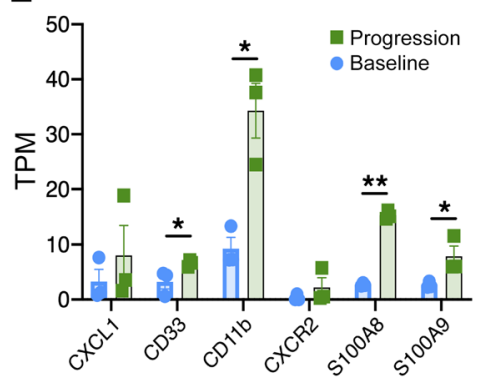

$\mathbf{F}$
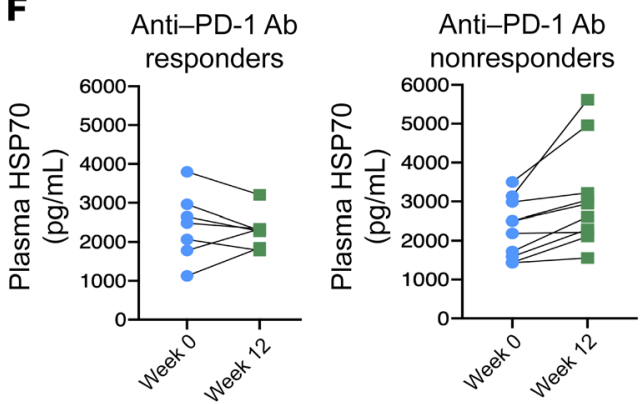

G

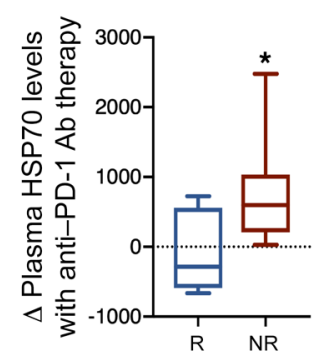

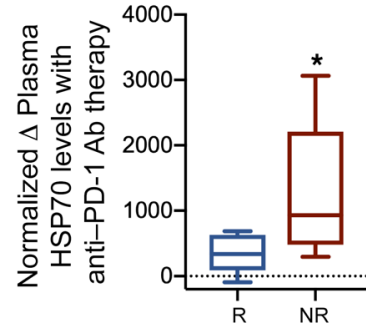

Figure 7. The PD-L1/NLRP3/HSP70 PMN-MDSC adaptive recruitment pathway in human melanoma. (A) Supernatant HSP70 and caspase-1 p20 Western blot analysis following treatment of human WM266 melanoma cells with IFN- $\gamma$ with or without anti-PD-L1 Ab. Blots are representative of 3 independent experiments. (B) Wnt5A Western blot analysis of HSP70-treated human WM266 melanoma cells with or without TLR4 inhibitor. Blots are representative of 3 independent experiments. (C) HSP70 and caspase-1 p20 Western blot analysis following treatment of human WM266 melanoma cells with ATP in the absence and presence of MCC950. Blots are representative of 2 independent experiments. (D) Cytolytic T cell markers correlated with ITCAM (CD11B), CD33, and NLRP3 gene expression in the melanoma TCGA-SKCM database. (E) RNA-Seq analysis of human melanoma tissue specimens collected before anti-PD-1 Ab therapy and at the time of disease progression on anti-PD-1 Ab therapy. TPM, transcripts per million. (F) Plasma HSP70 ELISA at week 0 and week 12 in patients with advanced melanoma undergoing anti-PD-1 Ab immunotherapy. (G) Change in HSP70 plasma levels following anti-PD-1 Ab immunotherapy in patients with advanced melanoma who were responders (R) or nonresponders (NR). The response was based on week-12 CT imaging. HSP70 changes were normalized to target tumor burden based on week-12 CT imaging. In the box-and-whisker plots, the central line represents the median, the box represents the first and third quartiles, and the error bars represent the data range. ${ }^{*} P<0.05$ and ${ }^{* *} P<0.005$, by Student's $t$ test (E and $\mathbf{G}$ ).

by tumor cells by an alternative, previously uncharacterized mechanism (43). Extracellular ATP has been implicated as a regulator of HSP70 release by tumor cells (32). Since ATP is a known modulator of the NLRP3 inflammasome, which in turn regulates the release of other inflammatory cytokines such as IL-1 $\beta$ and IL-18 that also lack leader peptide sequences, we initiated studies which confirmed that the NLRP3 inflammasome serves as an upstream regulator of HSP7O secretion by tumors (44). Indeed, these data demonstrate that both pharmacologic inhibition and genetic silencing of Nlrp3 effectively inhibited tumor release of HSP70 and ultimately eliminated the adaptive recruitment of PMNMDSCs in response to anti-PD- $1 \mathrm{Ab}$ therapy. It is important to note that we also conducted a series of experiments which showed no evidence that treatment with anti-PD-L1 Ab in vitro or anti$\mathrm{PD}-1 \mathrm{Ab}$ in vivo results in significant IL-1 $\beta$ expression or release in tumor cells or that IL- $1 \beta$ induces Wnt5a expression by tumor cells, as is observed with HSP70, thus suggesting that tumor- derived IL-1 $\beta$ does not contribute to MDSC recruitment in response to checkpoint inhibitor immunotherapy (Supplemental Figure 7, F-H) (45). This finding is also consistent with additional studies demonstrating that genetic knockout of HSP70 eliminated stimulation of Wnt5a in response to NLRP3 activation (Supplemental Figure 6B). Whether NLRP3-dependent IL-18 secretion contributes to the overall mechanism by promoting IFN- $\gamma$ expression is unclear and is currently being explored.

Using in vitro coculture assays and in vivo ablation experiments, these studies identified $\mathrm{CD} 8^{+} \mathrm{T}$ cell activity as an important driver for this tumor-intrinsic signaling pathway. This finding further prompted experiments which showed that IFN- $\gamma$ and its downstream modulation of PD-L1 were necessary for activation of the NLRP3 inflammasome. Although NF- $\mathrm{BB}$-dependent priming signals are required for the induction of NLRP3 activation in certain cell types such as macrophages, it appears that this signal was not necessary in the tumor models used here (33). Whether 
IFN- $\gamma$ stimulation also serves to facilitate NLRP3 priming by an alternative mechanism in tumor cells is currently being investigated. Interestingly, HSP7O/TLR4 signaling, as described above, may also provide a positive feed-forward priming pathway capable of perpetuating NLRP3 activation in tumors (46). These studies reveal that NLRP3 inhibition phenocopied downstream TLR4 and CXCR2 inhibition, suppressing the recruitment of PMN-MDSCs as an adaptive resistance mechanism initiated by local $\mathrm{CD}^{+} \mathrm{T}$ cell activity. This finding is in line with a previous study showing that NLRP3 can mitigate against DC vaccine therapies by promoting the migration of MDSCs into tumors (47).

Given the role of PD-L1 in the induction of this pathway as well as reports describing downstream signaling effects of PD-L1, we also conducted studies to better understand the underlying mechanism of NLRP3 activation in tumor cells (48). The previously described role of PKR in the regulation of inflammasome activation and its modulation by STAT3 led to a series of experiments culminating in our finding that PD-L1 induced PKR-mediated NLRP3 activation by inhibiting STAT3 $(34,35)$. Interestingly, these findings were consistent with those of other investigators who recently reported that the cytoplasmic domain of PD-L1 negatively regulates STAT3 in tumor cells (23). However, to our knowledge, this study is the first to report a mechanistic link between tumor PD-L1 and activation of the NLRP3 inflammasome in response to checkpoint inhibitor immunotherapy.

The overall findings of this study support further interrogation of this pathway as a source of both pharmacologic targets to augment the efficacy of immunotherapy and biomarkers to predict clinical responses and outcomes following exposure to immunotherapy. It should be noted that the use of CXCR2 inhibitors as a strategy to enhance anti-PD- 1 Ab immunotherapy is a concept that has been tested in preclinical tumor models and is currently being evaluated in early-phase clinical trials (Clinicaltrials.gov: NCT02583477, NCT03161431, NCT03473925) (8, 10). However, much like how PD-1/PD-L1 Ab antagonists offer a more tissueselective treatment approach over alternative immunotherapies, we conjecture that targeting the upstream NLRP3 inflammasome in this pathway will allow for more tumor-selective inhibition of CXCR2 chemokine-dependent recruitment of PMN-MDSCs, thus reducing the risk of systemic toxicity such as neutropenia. The NLRP3 target is also of particular interest in light of its described role in several inflammatory and autoimmune conditions, suggesting that the PD-L1/NLRP3 signaling axis may play an important role in driving some immune-related adverse events (irAEs) associated with checkpoint inhibitor immunotherapy (49, 50). These findings have prompted further study by our group to determine whether NLRP3 inhibition may mitigate against antiPD-1 Ab-induced irAEs and whether any genetic alterations of NLRP3 or its regulators may predict for the development of specific irAEs. Finally, it is tempting to speculate that there may be a potential relationship between this IFN- $\gamma$-dependent resistance pathway and the role of chronic IFN signaling in immunotherapy resistance described in a recent report (51).

In summary, we present evidence of an adaptive resistance signaling pathway that is inexorably linked to tumor PD-L1 and drives the recruitment of PMN-MDSCs to the tumor bed in response to anti-PD-1 checkpoint inhibitor therapy. This process extinguishes local cytotoxic antitumor $\mathrm{T}$ cell activity and serves as a rheostat for modulating effector $\mathrm{T}$ cell responses, thus making this signaling axis a promising target for immunotherapeutic intervention.

\section{Methods}

Clinical samples. All plasma samples were collected from 17 patients with advanced melanoma who were undergoing antiPD-1 Ab immunotherapy at week 0 and week 12 on an ongoing tissue acquisition protocol investigating checkpoint inhibitor resistance at Duke Cancer Institute (NCTO2694965). Three paired tumor specimens obtained from the patients at week 0 and at the time of disease progression while they were undergoing anti-PD-1 Ab immunotherapy were collected at Vanderbilt University Medical Center (institutional protocol no. 100178). Treatment responses were evaluated on the basis of RECIST (Response Evaluation Criteria in Solid Tumors, version 1.1).

In vivo animal studies. C57BL/6J (C57, $\left.\mathrm{H}-2^{\mathrm{b}}\right)$ (stock no. 000664), B6.CgBRAF ${ }^{\mathrm{tm} 1 \mathrm{Mmcm}} \mathrm{PTEN}^{\mathrm{tm} 1 \mathrm{Hwu}} \mathrm{Tg}$ (Tyr-Cre/ERT2)13Bos/ BosJ (BRAF ${ }^{\mathrm{V} 600 \mathrm{E}} \mathrm{PTEN}^{-/-}, \mathrm{H}-2^{\mathrm{b}}$ ) (stock no. 012328), and C57BL/ $6 \mathrm{Tg}$ (TcraTcrb) $1100 \mathrm{Mjb} / \mathrm{J}$ (OT-1, H-2 ${ }^{\mathrm{b}}$ ) (stock no. 003831) mice were obtained from The Jackson Laboratory. All experimental groups included randomly chosen 6- to 8-week-old littermate mice of both sexes and of the same strain.

Cell lines and culture conditions. BRAF ${ }^{\mathrm{V} 600 \mathrm{E}} \mathrm{PTEN}^{-/-}$(male, BPD6) (40), BRAF ${ }^{\mathrm{V} 600 \mathrm{E}} \mathrm{PTEN}^{-/-} \mathrm{Wnt}^{\mathrm{KD}}$ (40), $\mathrm{BRAF}^{\mathrm{V} 600 \mathrm{E}} \mathrm{PTEN}^{-/-}$ $\mathrm{CXCL5}^{\mathrm{KD}}, \mathrm{BRAF}^{\mathrm{V} 600 \mathrm{E}} \mathrm{PTEN}^{-/-} \mathrm{PDL1}^{\mathrm{KD}}, \mathrm{Braf}^{\mathrm{V}}{ }^{600 \mathrm{E}} \mathrm{PTEN}^{-/-} \mathrm{NLRP}^{\mathrm{KD}}$, and $\mathrm{BRAF}^{\mathrm{V} 600 \mathrm{E}} \mathrm{PTEN}^{-/-} \mathrm{NTC}(40)$ cell lines were generated using shRNA-expressing lentiviral vectors and cultured as previously described (40). The shRNA-expressing lentiviral vectors were as follows: shNLRP3 (MilliporeSigma, SHCLN-NM_145827), shPD-L1 (MilliporeSigma, SHCLND-NM_021893), shWnt5a (MilliporeSigma, SHCLND-NM_009524), shTLR4 (MilliporeSigma, SHCLNG-NM_025817), and pLKO.1-puro empty vector control (NTC) (MilliporeSigma, SHC001). The pEF.STAT3C.Ubc.GFP lentiviral expression plasmid (a gift from Kris Wood, Duke University) was used with the pMD2.G envelope vector and the psPAX2 packaging vector to produce lentiviral particles for generation of the $\mathrm{BRAF}^{\mathrm{V} 600 \mathrm{E}} \mathrm{PTEN}^{-/-} \mathrm{STAT}^{\mathrm{CA}}$ stable cell line. Stable cell lines were selected by puromycin resistance (MilliporeSigma, P8833). The OVA-expressing $\mathrm{BRAF}^{\mathrm{V} 600 \mathrm{E}} \mathrm{PTEN}^{-/-}$cell line was generated by transfecting the parental cell line with the pCI-neo-cOVA plasmid and selecting with $3 \mathrm{mg} / \mathrm{mL} \mathrm{G418}$. The murine LLC cell line was from the American Type Culture Collection (ATCC) (1704526). All cell lines were tested by the Duke University Cell Culture Facility shared services to ensure they were Mycoplasma free. All $\mathrm{BRAF}^{\mathrm{V} 600 \mathrm{E}} \mathrm{PTEN}^{-/-}$cell lines and the LLC cell line were maintained at $37^{\circ} \mathrm{C}$ in DMEM (Invitrogen, Thermo Fisher Scientific) with $2 \mathrm{mM} \mathrm{L-glutamine,} \mathrm{supplemented} \mathrm{with} 10 \% \mathrm{FBS}$ and $100 \mathrm{U} /$ $\mathrm{mL}$ penicillin. Depending on the experiment, cell lines were treated with Wnt5a (100-200 ng/mL, R\&D Systems, Bio-Techne, 645WN-010), IFN- $\gamma(100 \mathrm{ng} / \mathrm{mL}$, BioAbChem, 42-IFNg), anti-PD-L1 Ab (1-2 $\mu \mathrm{g} / \mathrm{mL})$, HSP70 (1-10 $\mu \mathrm{M}$, Enzo, ADI-ESP-502-D), HSP70 inhibitor (Thermo Fisher Scientific, VER155008), CLI-095 TLR4 inhibitor (3-10 $\mu \mathrm{M}$, Invivogen, tlrl-cli95), TLR2-IN-C29 TLR2 inhibitor (1-10 $\mu \mathrm{M}$, Glixx, GLXC-06203), MPLA TLR4 agonist (10 $\mu \mathrm{M}$, Enzo, ALX-581-205-C100), LPS (10 ng, MilliporeSigma, L4391-1MG), recombinant IL-1ß (100-200 ng, BioLegend, 
575102), ATP (1-5 mM, Invivogen, tlrl-atpl), NLRP3 inhibitor MCC950 (2.5-10 $\mu \mathrm{M}$, Invivogen, inh-mcc), XAV939 $\beta$-catenin inhibitor (0.5-1.0 $\mu \mathrm{M}$, MilliporeSigma, X3004-5MG), 2-aminopurine PKR inhibitor (1-5 mM, Invivogen, tlrl-apr), verteporfin YAP inhibitor (0.1-1 $\mu \mathrm{M}, \mathrm{R} \& \mathrm{D}$ Systems, Bio-Techne, 530510), or vehicle control either for 24 or 48 hours prior to in vitro and in vivo experiments. The control siRNA (sc-37007) and the TLR4 siRNA (sc-40261) were obtained from Santa Cruz Biotechnology.

Autochthonous tumor studies. B6.Cg-BRAF ${ }^{\mathrm{tm} 1 \mathrm{Mmcm}} \mathrm{PTEN}^{\mathrm{tm} 1 \mathrm{Hwu}}$ $\mathrm{Tg}$ (Tyr-Cre/ERT2 H-2 ${ }^{\mathrm{b}}$ )13Bos/BosJ (BRAF ${ }^{\mathrm{V} 600 \mathrm{E}} \mathrm{PTEN}^{-/-}$-transgenic) mice were subdermally injected with 4-hydroxytomoxifen (4-HT) (MilliporeSigma, H6278-50MG CCF; $38.75 \mu \mathrm{g} / \mathrm{mouse}$ ) to induce primary melanoma development at the base of the tail. Mice were randomly assigned to a treatment cohort once tumor volumes reached $64 \mathrm{~mm}^{3}(19,30,52)$. Depending on the experiment, the mice were treated with the following agents: CXCR2 inhibitor (AZD5069, AstraZeneca) at $100 \mathrm{mg} / \mathrm{kg}$ p.o. twice daily, NLRP3 inhibitor (MCC950) $10 \mathrm{mg} / \mathrm{kg}$ i.p. every other day, antiPD-1 Ab (Bio X Cell) or rat IgG2a isotype control (Bio X Cell) at $200 \mu \mathrm{g}$ by i.p. injection every 3 days, or dacarbazine $(50 \mathrm{mg} / \mathrm{kg}$ or $75 \mathrm{mg} / \mathrm{kg}$, MilliporeSigma, D2390) by i.p. injection once every other day. Melanoma growth was monitored by orthogonal caliper measurements every 3 days.

Syngeneic transplant tumor studies. BRAF ${ }^{\mathrm{V} 600 \mathrm{E}} \mathrm{PTEN}^{-/}$, $\mathrm{BRAF}^{\mathrm{V} 600 \mathrm{E}} \mathrm{PTEN}^{-/-} \mathrm{NTC}^{-} \mathrm{BRAF}^{\mathrm{V} 600 \mathrm{E}} \mathrm{PTEN}^{-/-} \mathrm{Wnt}^{5} \mathrm{a}^{\mathrm{KD}}, \mathrm{BRAF}^{\mathrm{V} 600 \mathrm{E}}$ $\mathrm{PTEN}^{-/-} \mathrm{CXCL}^{\mathrm{KD}}, \mathrm{BRAF}^{\mathrm{V} 600 \mathrm{E}} \mathrm{PTEN}^{-/-} \mathrm{PD}^{-\mathrm{L}^{\mathrm{KD}}}{ }^{\mathrm{K}}$, and BRAF ${ }^{\mathrm{V} 600 \mathrm{E}}$ $\mathrm{PTEN}^{-/-} \mathrm{NLRP}^{\mathrm{KD}}$ cell lines $\left(0.5 \times 10^{5}\right.$ to $1 \times 10^{5}$ cells $)$ were implanted by s.c. injection into the base of the tail of syngeneic C57BL/6 mice. Tumor growth was monitored by caliper measurement every 3 days, and treatment was initiated when tumor volumes reached $64 \mathrm{~mm}^{3}$. Tumor volume was calculated according to the formula: $\mathrm{cm}^{3}=\left[(\right.$ length, $\left.\mathrm{cm}) \times(\text { width }, \mathrm{cm})^{2}\right] / 2$.

Murine cell isolation. Tumors were resected and mechanically disaggregated by a gentleMACS dissociator (Miltenyi Biotec), filtered through $70-\mu \mathrm{m}$ filters, and digested with RPMI containing collagenase IV ( $1 \mathrm{mg} / \mathrm{mL}$, MilliporeSigma), hyaluronidase (0.1 mg/mL, MilliporeSigma), and deoxyribonuclease $(20 \mathrm{U} / \mathrm{mL}$, MilliporeSigma) on a shaker at $250 \mathrm{rpm}$ at $37^{\circ} \mathrm{C}$ for 1 hour (23). Resected splenic and lymph node tissues were mechanically disaggregated using 1-cc syringe plunger and $40-\mu \mathrm{m}$ filters followed by treatment with RBC lysis buffer (MilliporeSigma).

In vivo CD8 depletion. The hybridoma clone 53-6.7 was expanded at the Duke Cell Culture Facility in hollow fiber cartridges; 10 $\mathrm{mL}$ serum-free supernatant was harvested every 2 days. Antimouse CD8 Ab was purified using a Pierce Gentle Ag/Ab Binding and Elution Buffer Kit (Thermo Fisher Scientific, 21030) according to the manufacturer's protocol (Thermo Fisher Scientific). $\mathrm{Ab}$ concentration was determined by bicinchoninic acid (BCA) protein assay. Anti-CD8 Ab or IgG isotype control Ab was delivered daily for the first 3 days and then every 7 days thereafter by i.p. injection (500 $\mu \mathrm{g}$ /dose). For CD8 depletion of progressing $\mathrm{BRAF}^{\mathrm{V} 600 \mathrm{E}} \mathrm{PTEN}^{-/-} \mathrm{NTC}^{-}$and $\mathrm{BRAF}^{\mathrm{V} 600 \mathrm{E}} \mathrm{PTEN}^{-/-} \mathrm{NLRP}^{\mathrm{KD}}$ tumors, anti-CD8a Ab delivery was initiated when NLRP3 ${ }^{\mathrm{KD}}$ tumor growth became stagnant and followed the schedule described above. CD8 depletion was verified by splenocyte flow cytometric analysis.

In vitro tumor-killing assays. OT- $1 \mathrm{CD} 8^{+} \mathrm{T}$ cell activation was performed by incubating isolated splenocytes from OT-1-trans- genic mice with IL-2 $(100 \mathrm{U} / \mathrm{mL})$ and SIINFEKL peptide $(1 \mu \mathrm{g} / \mathrm{mL}$, New England Peptide, BP10-915) for 3 days followed by magnetic bead CD8 purification according to the manufacturer's instructions (Miltenyi Biotec, 130-104-075). Activated OT-1 CD8 ${ }^{+}$T cells were incubated with $\mathrm{BRAF}^{\mathrm{V} 600 \mathrm{E}} \mathrm{PTEN}^{-/-}$OVA cells and treated with antiPD-1 Ab $(1 \mu \mathrm{g} / \mathrm{mL})$ for 72 hours at a tumor cell/CD8 ${ }^{+} \mathrm{T}$ cell ratio of 1:5. In separate experiments, $\mathrm{BRAF}^{\mathrm{V} 600 \mathrm{E}} \mathrm{PTEN}^{-/-}$cells were treated with increasing concentrations of dacarbazine (10-400 $\mu \mathrm{mol})$.

IHC and immunofluorescence analysis. Paraffin-embedded tissues were processed and stained following standard protocols and imaged with a Zeiss CLSM 700 confocal microscope. The following Abs were used in IHC and immunofluorescence experiments: anti-Wnt5a $(1 \mu \mathrm{g} / \mathrm{mL})$, anti-Ly6G $(0.5 \mu \mathrm{g} / \mathrm{mL})$, anti-CD8a $(0.5 \mu \mathrm{g} / \mathrm{mL})$, and anti-CXCL5 $(1 \mu \mathrm{g} / \mathrm{mL})$. Tissue was permeabilized by incubation in $0.4 \%$ Triton-X in TBS for 20 minutes. Goat anti-rabbit Ab conjugated to Alexa Fluor 564 and goat anti-mouse $\mathrm{Ab}$ conjugated to Alexa Fluor 488 were used as secondary Abs for the appropriate primary Ab. For immunohistochemical analysis, anti-rat polymers were used as secondary Abs, and a Warp Red Chromogen Detection System (BioCare, WR806S) was used for antigen visualization.

Flow cytometric analysis. One million cells were stained with $1 \mu \mathrm{g}$ of each fluorochrome-conjugated $\mathrm{Ab}$ or commercially available dyes according to the standard protocols and analyzed using a FACSCanto II or LSR II (BD). Cells were incubated with $\mathrm{F}_{\mathrm{C}}$ receptor-blocking Abs followed by a live/dead discriminator (CellTrace Violet, Thermo Fisher Scientific, C34571) and then stained with conjugated $\mathrm{Abs}$ for 30 minutes at $4^{\circ} \mathrm{C}$. Cell numbers were calculated by hemocytometer, and flow cytometric data were analyzed using Flowjo software, version 10.3.

Immunoblotting, immunoprecipitation, and ASC polymerization. Tumor tissue or cells were homogenized in NP40 lysis buffer (MilliporeSigma) supplemented with complete protease inhibitor and phosphatase inhibitor (Roche). Cells were lysed in Laemmli sample buffer after treatment and subjected to SDS-PAGE. After transfer onto PVDF membranes (Bio-Rad), monoclonal and polyclonal primary Abs and appropriate HRP-conjugated secondary Abs were used for blotting. For the immunoprecipitation assays, cells were lysed with TBS buffer (50 mM Tris-HCl [pH 7.4], 150 $\mathrm{mM} \mathrm{NaCl}$ ) containing $0.5 \%$ Triton X-100, EDTA-free protease inhibitor cocktail, and phosphatase inhibitor cocktail, precleared with protein A/G beads, then incubated with $1 \mu \mathrm{g}$ of the appropriate $\mathrm{Abs}$ or isotype control IgG on a rotator overnight at $4^{\circ} \mathrm{C}$ and then with protein $\mathrm{A} / \mathrm{G}-$ agarose at $4^{\circ} \mathrm{C}$ for 1 hour. After 5 washes with TBS buffer, immunoprecipitated complexes were eluted in sample buffer by boiling and then subjected to immunoblot analysis. Immunoblots were visualized by chemiluminescent substrate (Thermo Fisher Scientific) and imaged with a ChemiDoc XRSplus system (Bio-Rad). For the ASC polymerization assay, cells were lysed with TBS buffer containing 0.5\% Triton X-100, EDTA-free protease inhibitor cocktail, and phosphatase inhibitor cocktail. The lysates were centrifuged at $4000 \mathrm{~g}$ at $4^{\circ} \mathrm{C}$ for 15 minutes. For the detection of ASC oligomerization, the Triton-insoluble pellets were washed twice with TBS buffer and then resuspended in 500 $\mu \mathrm{L}$ TBS buffer. The resuspended pellets were crosslinked for 30 minutes at room temperature with $2 \mathrm{mM}$ disuccinimidyl suberate (DSS) (Thermo Fisher Scientific, 21655) and then centrifuged for 
15 minutes at $6000 \mathrm{~g}$. The pellets were dissolved in SDS sample buffer. See complete unedited blots in the supplemental material.

Abs. The following Abs were used: anti- $\beta$-actin, mouse $\mathrm{mAb}$ (Santa Cruz Biotechnology, sc-47778); anti-NLRP3, rabbit mAb (Cell Signaling Technology, 15101S); anti-ASC, mouse mAb (Santa Cruz Biotechnology, sc-514414); anti-caspase-1 p20, mouse mAb (Adipogen, AG-20B-0042-C100); anti-caspase-3, rabbit polyclonal Ab (Cell Signaling Technology, 9662S); anti-HSP70, mouse mAb (Santa Cruz Biotechnology, sc-66048); anti-CXCL5, goat polyclonal Ab (R\&D Systems, AF433); anti-CXCL5, rabbit polyclonal Ab (LSBio, LS-c293780); anti-YAP/TAZ, rabbit mAb (Cell Signaling Technology, 8418S); anti-Wnt5a, mouse mAb (Santa Cruz Biotechnology, sc-365370); anti-GAPDH, mouse mAb (Santa Cruz Biotechnology, sc-32233); CD8a, rabbit mAb (Cell Signaling Technology, 989415); InVivoMAb anti-human PD-L1 Ab (Bio X Cell, BE0285); InVivoMAb anti-mouse PD-L1 Ab (Bio X Cell, BE0101); InVivoMAb anti-mouse PD-1 Ab (Bio X Cell, BE0146); InVivoMAb rat IgG2a isotype control Ab, clone: $2 \mathrm{~A} 3$ (Bio X Cell, BE0089); anti-mouse CD8 Ab from hybridoma, (Duke Cell Culture Facility, clone 53.6.7); anti-OVA Ab (Santa Cruz Biotechnology, sc-65984); anti-IL-1 $\beta$, mouse mAb (Cell Signaling Technology, 12242); anti-LY6G-Gr1 Ab (Abcam, ab25377); goat anti-rat IgG $\mathrm{H} \& \mathrm{~L}$ Ab (MilliporeSigma, AP136P).; IFN- $\gamma$, mouse mAb (Novus Biologicals, MAB4851-SP); anti-STAT3 Ab, mouse mAb (Santa Cruz Biotechnology, sc-8019); anti-phosphorylated STAT3 (anti-p-STAT3) Ab, mouse monoclonal (Cell Signaling Technology, 9131); anti-PKR Ab, mouse mAb (Santa Cruz Biotechnology, sc-6282); anti-p-PKR (Thr446) Ab, rabbit mAb (Abcam, ab32036); anti-vinculin $\mathrm{Ab}$, rabbit $\mathrm{mAb}$ (Invitrogen, Pierce, Thermo Fisher Scientific, 700062); anti-laminin b1 Ab, mouse mAb (Santa Cruz Biotechnology, Sc-374015); anti-mouse CD11c, FITC-conjugated, clone: HL3 (BD Pharmingen, 553801); anti-mouse CD11b, PE-conjugated, clone: MIH5 (BD Pharmingen, 558091); antimouse CD8a Ab, BV510-conjugated, clone: 53-6.7 (BD Pharmingen, 563068); anti-mouse CD3e Ab, PerCP-Cy5.5-conjugated, clone: 145-2C11 (BD Pharmingen, 551163); anti-mouse Ly6GGR1 Ab, FITC-conjugated, clone: RB6-8C5 (BD Pharmingen, 5532127; anti-mouse F4/80 Ab, APC-conjugated, clone: BM8 (BD Pharmingen, 560408); anti-mouse CD45 Ab, PerCP-Cy5.5-conjugated, clone: 145-2C11 (BD Pharmingen, 551163); anti-mouse Ly6C Ab, PE-Cy7-conjugated, clone: AL-21 (BD Pharmingen, 560593); anti-human HLA-DR Ab, PerCP-Cy5.5-conjugated, clone: tu36 (BioLegend, 361607); anti-human CD15 Ab, PE-Cy7conjugated, clone: h198 (BD Pharmingen, 560827); anti-human CD33 Ab, Bv510-conjugated, clone: wm53 (BD Pharmingen, 563257); anti-human CD11b Ab, PE-conjugated (BD Pharmingen, 557321); and anti-human CD14 Ab, FITC-conjugated (BD Pharmingen, 557153).

RNA isolation and $q R T-P C R$ analysis. Total RNA was isolated using the RNeasy Plus Mini Kit (QIAGEN, 74134). RNA (1000 ng) was used for cDNA synthesis (iScript Reverse Transcription Supermix, Bio-Rad, 1708841). qRT-PCR was performed using an ABI7500 Real-Time PCR system (Life Technologies, Thermo Fisher Scientific). The primers used are listed in Supplemental Table 1. Data analysis was performed using PrimePCR Analysis Software (Bio-Rad). Conventional qRT-PCR was performed using validated primers and SsoAdvanced Universal SYBR Green
Supermix (Bio-Rad, 1725271) or SsoAdvanced Universal Probes Supermix (Bio-Rad, 1725281). All data were normalized to Actb expression, and relative gene expression was quantitated using the $2 \Delta \Delta \mathrm{Ct}$ method.

RNA-Seq assays. RNA-Seq on tumor tissue derived from the BRAF $^{\mathrm{V} 600 \mathrm{E}} \mathrm{PTEN}^{-/-}$-transgenic murine model was performed by the Duke Sequencing and Genomic Technologies Shared Resource and the results previously published (30). A complementary DNA library was prepared via oligo-dT-directed reverse transcription (Ambion, Thermo Fisher Scientific) and subjected to deep sequencing on an Illumina HiSeq 4000 (50-bp single-read sequencing; Anti-PD-1 Resistance Study RNA-Seq, accession no. SAMN09878780). These data were processed by the Duke Center for Genomic and Computational Biology with the Trim Galore tool kit, which uses Cutadapt to trim low-quality bases and Illumina sequencing adapters from the $3^{\prime}$ end of the reads. Only reads that were $20 \mathrm{nt}$ or longer after trimming were kept for further analysis. Reads were mapped to the GRCm38v68 version of the mouse genome and transcriptome using the STAR RNA-Seq alignment tool (https://code.google.com/archive/p/rna-star/). Reads were kept for subsequent analysis if they mapped to a single genomic location. Gene counts were compiled using the HTSeq tool (https://pypi.org/project/HTSeq/). Only genes that had at least 10 reads in any given library were used in subsequent analyses. Normalization and differential expression were carried out using the DESeq2 Bioconductor package with the $\mathrm{R}$ statistical programming environment (https://bioconductor.org/packages/ release/bioc/html/DESeq.html). The FDR was calculated to control for multiple hypotheses testing. Gene set enrichment analysis (GSEA) was performed to identify differentially regulated pathways and Gene Ontology (GO) terms for each of the comparisons performed. Human melanoma tissues obtained from Vanderbilt University were also subjected to RNA-Seq analysis, and these data were previously published and deposited at https://prod.tbilab.org/balko_lab/LAG3_JCI_Insight (53).

ELISA. Levels of CXCL5 and HSP70 (R\&D Systems, DYC16632) in mouse plasma were evaluated using an ELISA kit according to the manufacturer's instructions (R\&D Systems). IL-1 $\beta$ (BioLegend, 432601) levels in cell lysate, tumor lysate, and supernatant were measured using the BioLegend ELISA kit according to the manufacturer's instructions.

Human plasma ELISA. Human plasma HSP70 concentrations were measured using the human DuoSet Assay (R\&D Systems, catalog DY1663) according to the manufacturer's protocol. Human melanoma plasma samples were obtained from the Duke Cancer Institute.

Secretome assays. Single-cell suspensions were prepared by enzymatic and mechanical digestion. Cells $\left(2 \times 10^{6}\right)$ were plated in $2 \mathrm{~mL} \mathrm{1 \%}$ dialyzed FBS containing light or heavy amino acids without L-methionine in 6-well plates and then incubated in $\mathrm{CO}_{2}$ for 30 minutes. L-methionine or AHA, an azide-bearing analog of methionine, was added to the plates and incubated in $5 \% \mathrm{CO}_{2}$ at $37^{\circ} \mathrm{C}$ overnight. Culture supernatants were collected for a secretome assay. Cell supernatants were enriched for AHA-labeled proteins by incubating with dibenzocyclooctyne (DBCO) agarose overnight. Resins were washed, followed by reduction and alkylation of cysteine (Cys) residues, and peptides were recovered after 
overnight digestion with trypsin. Samples were analyzed by quantitative 1-dimensional LC-MS/MS. Using Proteome Discoverer 2.3, the data were searched against the SwissPro Mouse database with semitrypsin specificity, fixed modification on Cys, and variable modifications on Met (oxidation), Gln/Asn (deamidation), Pro (hydroxylation), and Arg/Lys (15N-13C).

TCGA data analysis. TCGA skin cancer melanoma (TCGASKCM) annotated RNA expression files were extracted from the GDC portal (https://portal.gdc.cancer.gov/) for 376 cases of metastatic melanoma. Data preprocessing was performed using HTSeq counts from TCGA, and raw counts were normalized using the relative log expression method implanted in $\mathrm{R}$ and its extension package DESeq2. Coexpression of genes of interest were tested using the $\chi^{2}$ statistic of sample quantile-based contingency (SQUAC) table, and scatter plots were generated using normalized counts and Bonferroni's corrections on the group $P$ values to account for multiple testing.

Statistics. GraphPad Prism 8 for Windows (GraphPad Software) was used for all statistical analyses. An unpaired, 2-tailed Student's $t$ test was used to compare mean differences between the control and treatment groups. Univariate 1-way ANOVA followed by Sidak's post hoc multiple comparisons test was performed to analyze data containing 3 or more groups. Data correlation analyses were conducted using either Kendall Tau or Spearman calculations. A $P$ value of less than 0.05 was considered significant. All quantitative data are presented as the mean \pm SEM.

Study approval. Mouse tumor experiments were performed according to a protocol approved by the IACUC of Duke University Medical Center. All patients provided written informed consent under approval from the IRBs of Duke University (NCT02694965) and Vanderbilt University Medical Center (protocol 100178). Human melanoma tissues were obtained from Vanderbilt University with IRB approval (protocol 100178). Human melanoma plasma samples were obtained from the Duke Cancer Institute with IRB approval (protocol Pro00059349, Clinicaltrials.gov: NCT02694965).

\section{Author contributions}

BT and BAH conceptualized the project, designed all experiments, and analyzed all data. KSE, M. Sturdivant, M. Starr, LPW, YK, and DH provided technical support. AKSS, JMB, and DBJ provided clinical resources for the project. $\mathrm{BAH}$ and $\mathrm{ABN}$ supervised all experiments. BAH wrote the manuscript. BAH, AH, BT, NCD, and MP reviewed and edited the manuscript.

\section{Acknowledgments}

We would like to thank Michael Cook with the Duke Flow Cytometry Core, Matthew Foster with the Duke Proteomics Core, and Kouros Owzar with the Duke Biostatistics Core. This work was supported in part by the NIH (K08 CA191063-04, to BAH); a Melanoma Research Alliance Pilot Award (to BAH); the Ross Bierkan Melanoma Fund (to BAH and AKSS); a Duke University Health Scholar Award (to BAH); a Merck \& Co. Pre-Clinical Award (to $\mathrm{BAH}$ ); and an AstraZeneca Pre-Clinical Award (to BAH).

Address correspondence to: Brent A. Hanks, 308 Research Drive, LSRC, C203, Box 91004, Duke University, Durham, North Carolina 27708, USA. Phone: 919.684.1995; Email: brent.hanks@duke.edu.
1. Zhao X, Subramanian S. Intrinsic resistance of solid tumors to immune checkpoint blockade therapy. Cancer Res. 2017;77(4):817-822.

2. Pitt JM, et al. Resistance mechanisms to immune-checkpoint blockade in cancer: tumor-intrinsic and -extrinsic factors. Immunity. 2016;44(6):1255-1269.

3. O'Donnell JS, Long GV, Scolyer RA, Teng MW, Smyth MJ. Resistance to PD1/PDL1 checkpoint inhibition. Cancer Treat Rev. 2017;52:71-81.

4. Marigo I, Dolcetti L, Serafini P, Zanovello P, Bronte V. Tumor-induced tolerance and immune suppression by myeloid derived suppressor cells. Immunol Rev. 2008;222:162-179.

5. Marvel D, Gabrilovich DI. Myeloid-derived suppressor cells in the tumor microenvironment: expect the unexpected. J Clin Invest. 2015;125(9):3356-3364.

6. Sade-Feldman M, et al. Clinical significance of circulating $\mathrm{CD}^{2} 3^{+} \mathrm{CD} 11 \mathrm{~b}^{+} \mathrm{HLA}-\mathrm{DR}$ - myeloid cells in patients with stage IV melanoma treated with ipilimumab. Clin Cancer Res. 2016;22(23):5661-5672.

7. Weber J, et al. Phase I/II study of metastatic melanoma patients treated with nivolumab who had progressed after ipilimumab. Cancer Immunol Res. 2016;4(4):345-353.

8. Highfill SL, et al. Disruption of CXCR2-mediated MDSC tumor trafficking enhances anti-PD1 efficacy. Sci Transl Med. 2014;6(237):237ra67.

9. Chao T, Furth EE, Vonderheide RH. CXCR2dependent accumulation of tumor-associated neutrophils regulates T-cell immunity in pancreatic ductal adenocarcinoma. Cancer Immunol Res. 2016;4(11):968-982.

10. Steele CW, et al. CXCR2 inhibition profoundly suppresses metastases and augments immunotherapy in pancreatic ductal adenocarcinoma. Cancer Cell. 2016;29(6):832-845.

11. Sharma P, Hu-Lieskovan S, Wargo JA, Ribas A. Primary, adaptive, and acquired resistance to cancer immunotherapy. Cell. 2017;168(4):707-723.

12. Neubert NJ, et al. T cell-induced CSF1 promotes melanoma resistance to PD1 blockade. Sci Transl Med. 2018;10(436):eaan3311.

13. Chen L, et al. CD38-mediated immunosuppression as a mechanism of tumor cell escape from PD-1/PD-L1 Blockade. Cancer Discov. 2018;8(9):1156-1175.

14. Munn DH, Mellor AL. Indoleamine 2,3-dioxygenase and tumor-induced tolerance. JClin Invest. 2007;117(5):1147-1154.

15. Spranger S, et al. Up-regulation of PD-L1, IDO, and $\mathrm{T}$ (regs) in the melanoma tumor microenvironment is driven by CD8(+) T cells. Sci Transl Med. 2013;5(200):200ra116.

16. Li J, et al. Tumor cell-intrinsic factors underlie heterogeneity of immune cell infiltration and response to immunotherapy. Immunity. 2018;49(1):178-193.e7.

17. Anastas JN, et al. WNT5A enhances resistance of melanoma cells to targeted BRAF inhibitors. J Clin Invest. 2014;124(7):2877-2890.
18. Hugo W, et al. Genomic and transcriptomic features of response to anti-PD-1 therapy in metastatic melanoma. Cell. 2016;165(1):35-44.

19. Zhao F, et al. Paracrine Wnt5a- $\beta$-catenin signaling triggers a metabolic program that drives dendritic cell tolerization. Immunity. 2018;48(1):147-160.e7.

20. Blumenthal A, et al. The wingless homolog WNT5A and its receptor Frizzled-5 regulate inflammatory responses of human mononuclear cells induced by microbial stimulation. Blood. 2006;108(3):965-973.

21. Oblak A, Jerala R. Toll-like receptor 4 activation in cancer progression and therapy. Clin Dev Immunol. 2011;2011:609579.

22. Dong P, Xiong Y, Yue J, Hanley SJB, Watari H. Tumor-intrinsic PD-L1 signaling in cancer initiation, development and treatment: beyond immune evasion. Front Oncol. 2018;8:386.

23. Gato-Cañas M, et al. PDL1 signals through conserved sequence motifs to overcome interferon-mediated cytotoxicity. Cell Rep. 2017;20(8):1818-1829.

24. Escors D, et al. The intracellular signalosome of PD-L1 in cancer cells. Signal Transduct Target Ther. 2018;3:26.

25. Moossavi M, Parsamanesh N, Bahrami A, Atkin SL, Sahebkar A. Role of the NLRP3 inflammasome in cancer. Mol Cancer. 2018;17(1):158.

26. Soler-Cardona A, et al. CXCL5 facilitates melanoma cell-neutrophil interaction and lymph node metastasis. J Invest Dermatol. 
2018;138(7):1627-1635.

27. Park HW, et al. Alternative Wnt signaling activates YAP/TAZ. Cell. 2015;162(4):780-794.

28. Wang G, et al. Targeting YAP-dependent MDSC infiltration impairs tumor progression. Cancer Discov. 2016;6(1):80-95.

29. Asea A, et al. Novel signal transduction pathway utilized by extracellular HSP70: role of tolllike receptor (TLR) 2 and TLR4. J Biol Chem. 2002;277(17):15028-15034.

30. Zhao F, et al. Stromal fibroblasts mediate antiPD-1 resistance via MMP-9 and dictate TGF $\beta$ inhibitor sequencing in melanoma. Cancer Immunol Res. 2018;6(12):1459-1471.

31. Eichelbaum K, Winter M, Berriel Diaz M, Herzig S, Krijgsveld J. Selective enrichment of newly synthesized proteins for quantitative secretome analysis. Nat Biotechnol. 2012;30(10):984-990.

32. Mambula SS, Calderwood SK. Heat shock protein 70 is secreted from tumor cells by a nonclassical pathway involving lysosomal endosomes. JImmunol. 2006;177(11):7849-7857.

33. Swanson KV, Deng M, Ting JP. The NLRP3 inflammasome: molecular activation and regulation to therapeutics. Nat Rev Immunol. 2019;19(8):477-489.

34. Lu B, et al. Novel role of PKR in inflammasome activation and HMGB1 release. Nature. 2012;488(7413):670-674.

35. Shen S, et al. Cytoplasmic STAT3 represses autophagy by inhibiting PKR activity. Mol Cell. 2012;48(5):667-680.

36. Bai H, Zhang QF, Duan JJ, Yu DJ, Liu LJ. Down- regulation of signal transduction and STAT3 expression exacerbates oxidative stress mediated by NLRP3 inflammasome. Neural Regen Res. 2018;13(12):2147-2155.

37. Coll RC, et al. A small-molecule inhibitor of the NLRP3 inflammasome for the treatment of inflammatory diseases. Nat Med. 2015;21(3):248-255.

38. Ndoye A, et al. ATG5 mediates a positive feedback loop between Wnt signaling and autophagy in melanoma. Cancer Res. 2017;77(21):5873-5885.

39. Da Forno PD, et al. WNT5A expression increases during melanoma progression and correlates with outcome. Clin Cancer Res. 2008;14(18):5825-5832.

40. Holtzhausen A, et al. Melanoma-derived Wnt5a promotes local dendritic-cell expression of IDO and immunotolerance: opportunities for pharmacologic enhancement of immunotherapy. Cancer Immunol Res. 2015;3(9):1082-1095.

41. Fang H, et al. Toll-like receptor 4 (TLR4) is essential for Hsp70-like protein 1 (HSP70L1) to activate dendritic cells and induce Th1 response. J Biol Chem. 2011;286(35):30393-30400.

42. Murphy ME. The HSP7O family and cancer. Carcinogenesis. 2013;34(6):1181-1188.

43. Radons J. The human HSP7O family of chaperones: where do we stand? Cell Stress Chaperones. 2016;21(3):379-404.

44. He Y, Hara H, Núñez G. Mechanism and regulation of NLRP3 inflammasome activation. Trends Biochem Sci. 2016;41(12):1012-1021.

45. Tu S, et al. Overexpression of interleukin-1beta induces gastric inflammation and cancer and mobilizes myeloid-derived suppressor cells in mice. Cancer Cell. 2008;14(5):408-419.

46. Qu J, et al. Blocking ATP-sensitive potassium channel alleviates morphine tolerance by inhibiting HSP70-TLR4-NLRP3-mediated neuroinflammation. J Neuroinflammation. 2017;14(1):228.

47. van Deventer HW, et al. The inflammasome component NLRP3 impairs antitumor vaccine by enhancing the accumulation of tumor-associated myeloid-derived suppressor cells. Cancer Res. 2010;70(24):10161-10169.

48. Lecis D, Sangaletti S, Colombo MP, Chiodoni C. Immune checkpoint ligand reverse signaling: looking back to go forward in cancer therapy. Cancers (Basel). 2019;11(5):E624.

49. Cordero MD, Alcocer-Gómez E, Ryffel B. Gain of function mutation and inflammasome driven diseases in human and mouse models. JAutoimmun. 2018;91:13-22.

50. Booshehri LM, Hoffman HM. CAPS and NLRP3. JClin Immunol. 2019;39(3):277-286.

51. Benci JL, et al. Tumor interferon signaling regulates a multigenic resistance program to immune checkpoint blockade. Cell. 2016;167(6):1540-1554.e12.

52. Holtzhausen A, et al. Melanoma-derived Wnt5a promotes local dendritic-cell expression of IDO and immunotolerance: opportunities for pharmacologic enhancement of immunotherapy. Cancer Immunol Res. 2015;3(9):1082-1095.

53. Johnson DB, et al. Tumor-specific MHC-II expression drives a unique pattern of resistance to immunotherapy via LAG-3/FCRL6 engagement. JCI Insight. 2018;3(24):e120360. 